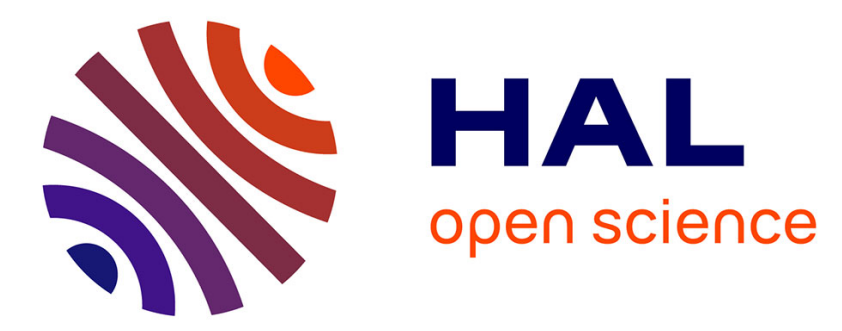

\title{
Pair-particle trajectories in a shear flow of a Bingham fluid
}

\author{
Hassan Fahs, Guillaume Ovarlez, Xavier Chateau
}

\section{To cite this version:}

Hassan Fahs, Guillaume Ovarlez, Xavier Chateau. Pair-particle trajectories in a shear flow of a Bingham fluid. Journal of Non-Newtonian Fluid Mechanics, 2018, 261, pp.171-187. 10.1016/j.jnnfm.2018.07.010 . hal-01982895

\section{HAL Id: hal-01982895 \\ https://hal.science/hal-01982895}

Submitted on 17 Jan 2019

HAL is a multi-disciplinary open access archive for the deposit and dissemination of scientific research documents, whether they are published or not. The documents may come from teaching and research institutions in France or abroad, or from public or private research centers.
L'archive ouverte pluridisciplinaire HAL, est destinée au dépôt et à la diffusion de documents scientifiques de niveau recherche, publiés ou non, émanant des établissements d'enseignement et de recherche français ou étrangers, des laboratoires publics ou privés. 


\title{
Pair-particle trajectories in a shear flow of a Bingham fluid
}

\author{
Hassan Fahs ${ }^{\mathrm{a}}$, Guillaume Ovarlez ${ }^{\mathrm{b}}$, Xavier Chateau ${ }^{\mathrm{a}, *}$ \\ a NAVIER, UMR 8205, École des Ponts, IFSTTAR, CNRS, UPE, Champs-sur-Marne, France \\ ${ }^{\mathrm{b}}$ University of Bordeaux, CNRS, Solvay, LOF, UMR 5258, Pessac F-33608, France
}

\section{A B $S$ T R A C $T$}

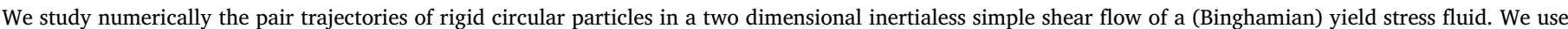

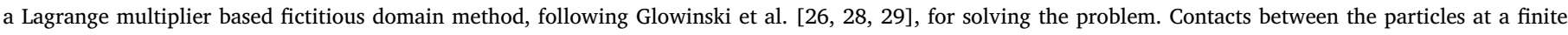

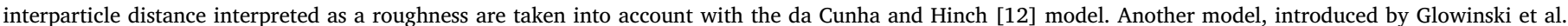

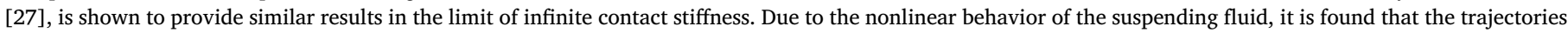

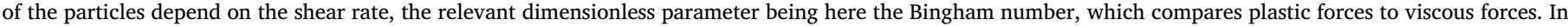

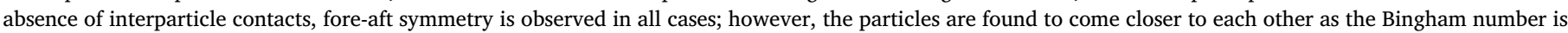

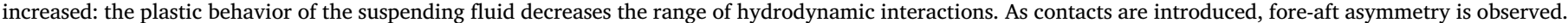

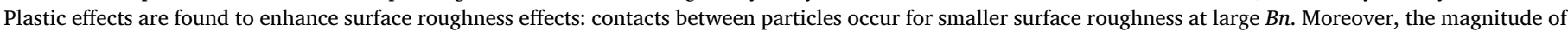

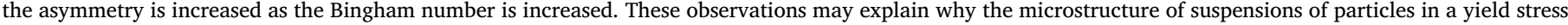
fluid is shear-rate-dependent [45] leading to a complex nonlinear macroscopic behavior.

\section{Introduction}

Suspensions of non-Brownian and noncolloidal hard spheres are relevant model systems to help understanding the behavior of complex polydisperse suspensions found in the industry (fresh concrete...) and in geophysics (debris-flows...) [1,11,20,25,36,40,55].

Substantial progress in the understanding of the behavior of such materials can thus be made by studying the impact of adding noncolloidal particles to a yield stress fluid of known properties [8,13,39,45,57]. From a more fundamental point of view, these systems, viewed as rigid inclusions in a nonlinear material, may provide crucial tests for micromechanical approaches developed to describe the behavior of suspensions and composite materials.

A key element in the understanding and modeling of the macroscopic behavior of suspensions is the hydrodynamic interaction between particles and its impact on the suspensions' microstructure [1,7]. Many information can readily be obtained by studying a seemingly simple problem involving interactions between a single pair of particles, that is, the study of the relative trajectories of two particles in a simple shear flow.

The case of particles in an inertialess Newtonian fluid has been studied in much detail $[30,43]$. For perfectly smooth particles, two families of trajectories exist. At close interparticle distance, particles are found to orbit around each other; otherwise, they cross each other by following a symmetric trajectory in the frame centered on their neighbor, which comes from the linearity of the Stokes equation and the symmetries of the problem. Such symmetry is not observed experimentally for nonperfectly smooth particles [49]. This has been attributed to the finite

\footnotetext{
* corresponding author.

E-mail address: xavier.chateau@enpc.fr (X. Chateau).
}

roughness of the particles, which introduces a cut-off in the hydrodynamic interaction between the particles, which now makes the problem nonlinear. Consequently, the fore-aft symmetry is broken. It is finally found that the modeling of the trajectories based on the experimentally measured roughness allows to describe accurately experimental results [6]. At the scale of suspensions, this induces asymmetric pair distributions functions (pdfs) and nonzero normal stress differences [43]. Again, when the experimentally measured roughness is taken into account to model the hydrodynamic interactions between pairs of particles, very good agreement is found between the experimentally measured and numerically computed pdfs [5].

The study of particles in Newtonian fluids has been extended to inertial flows $[30,31,38]$. In this last case, the fore-aft symmetry is also found to be broken, due to the nonlinear convective acceleration; the magnitude of the asymmetry then depends on the Reynolds number. This has been found to be accompanied with the vanishing of closed particle trajectories [38], and to the emergence of new forms of trajectories. All these features are predicted to impact the rheological behavior of inertial suspensions, and to lead to observable Reynolds number dependent rheological properties [31].

Particles in non-Newtonian fluids have been much less studied, although the case of particles suspended in viscoelastic fluids have received a lot of attention recently, in particular because of the tendency of particles to form chains under flow [15]. Since yield stress fluids are used in the industry to stabilize the particles against gravity, the sedimentation problem has been the subject of most studies on particles suspended in yield stress fluids $[4,22,46,53]$, with a focus on a single particle and on the onset of flow. The rheological properties of model 
suspensions have also been studied recently experimentally $[13,39,45]$ and theoretically $[3,8]$. The method used by Chateau et al. [8], valid for any generalized Newtonian behavior, consists, first, in assuming that the overall properties of the suspension can be accurately estimated from an average estimate $\bar{\gamma}_{\text {local }}$ of the local shear rate over the suspending fluid domain, second, in using one of the material properties determined experimentally to estimate the value of $\bar{\gamma}_{\text {local }}$, and finally, in using this last value to predict the other properties. Estimates of the suspension apparent viscosity have then been obtained by linearizing the suspending fluid behavior at each prescribed macroscopic shear rate and computing its effective viscosity at the average local shear rate $\overline{\dot{\gamma}}_{\text {local }}$. A major result is then the interdependence of the evolutions of all rheological properties with the particle volume fraction: it seems that all rheological properties can be predicted as soon as one of them is known. This has first been validated experimentally on isotropic suspensions by Mahaut et al. [39]. However, some discrepancy between the rheological properties evolutions observed at low and high shear rates was reported by Ovarlez et al. [45] for sheared suspensions. This discrepancy was attributed to the emergence of a shear-rate-dependent microstructure for the suspensions, as observed experimentally; it was indeed pointed out that the interdependence between rheological properties at low and high shear rate is obtained theoretically only under the assumption that the microstructure is shear-rate-independent. The observed shear-rate-dependent microstructure can have a large impact on the rheological behavior; e.g., it was found that the stress at flow onset is strongly dependent on shear history.

To better understand the origin of a shear-rate-dependent microstructure in yield stress fluids, we study numerically the hydrodynamic interaction of two particles in an inertialess simple shear flow of a (Binghamian) yield stress fluid in two dimensions (2D). Pair trajectories are computed by solving a Lagrange multiplier based fictitious domain method, following Glowinski et al. [26, 28, 29]. Contacts between the particles at a finite interparticle distance interpreted as a roughness are taken into account with the da Cunha and Hinch [12] model. Another model, introduced by Glowinski et al. [27], is shown to provide similar results in the limit of infinite contact stiffness.

In Section 2, we first present precisely the studied problem. The numerical method used to solve the problem is then described in detail in Section 3. The results are finally shown and discussed in Section 4 before conclusions.

\section{Mathematical model}

We study the interaction of two neutrally buoyant and equal-sized circular particles in $\mathbb{R}^{2}$ suspended in an incompressible Bingham fluid undergoing a simple shear flow of shear rate $\dot{\gamma}$. In the simulations, we approximate this problem by solving the equations in a box with boundary conditions chosen to ensure that the average shear rate is $\dot{\gamma}$. It is then expected that, for large boxes, this might provide a good approximation of the solution for an infinite domain.

A sketch of the two-dimensional problem that we solve is shown in Fig. 1: two particles of radius $a$, denoted by $P_{1}(t)$ and $P_{2}(t)$, are placed symmetrically in a rectangular box, $\Omega$, of size $L \times W$. The collective particle region at a certain time $t$ is denoted by $P(t)=P_{1}(t) \cup P_{2}(t)$. External boundaries are denoted by $\Gamma=\partial \Omega=\bigcup_{i=1}^{4} \Gamma_{i}$ and the boundary of the $i$ th particle by $\partial P_{i}(t)$. The origin of the coordinate system is located at the center of the domain; $x$ is the flow direction and $y$ is the velocity gradient direction. On the upper and lower boundaries, equal and opposite velocities, $\pm \boldsymbol{U}_{\mathrm{W}}= \pm \dot{\gamma} W / 2$, are prescribed. Periodic boundary conditions are prescribed on the left and right boundaries. We refer to the initial perpendicular distance between the line of motion and the $x$-axis as the initial offset and denote it by $y_{-\infty}$ (e.g., it corresponds to the far upstream $y$-coordinate of $P_{1}(t)$, as $\left.x \rightarrow-\infty\right)$. Changing the value of the parameter $y_{-\infty}$ allows to tune the strength of hydrodynamic interaction between the two particles. We neglect inertia and body forces for both the fluid and the particles.

Hence, the motion of the fluid is governed by the Stokes set of equations

$\boldsymbol{\nabla} \cdot \boldsymbol{\sigma}=0$ in $\Omega \backslash P(t)$,

$\boldsymbol{\nabla} \cdot \boldsymbol{u}=0$ in $\Omega \backslash P(t)$,

where $\boldsymbol{u}$ is the fluid velocity and $\boldsymbol{\sigma}$ is the total stress tensor. Eqs. (1) and (2) are the balance equations of momentum and mass, respectively. For a viscoplastic Bingham fluid, $\sigma$ is split into spherical and deviatoric parts:

$\sigma=-p \boldsymbol{I}+\boldsymbol{\tau}$,

where $p$ is the pressure, $\boldsymbol{I}$ is the second-order identity tensor, and $\tau$ is the deviatoric stress tensor.

The constitutive law for the Bingham fluid reads

$\begin{array}{ll}\boldsymbol{\tau}(\boldsymbol{u})=2 \eta \boldsymbol{D}(\boldsymbol{u})+\tau_{0} \frac{\boldsymbol{D}(\boldsymbol{u})}{|\boldsymbol{D}(\boldsymbol{u})|} & \text { if }|\boldsymbol{D}(\boldsymbol{u})| \neq 0, \\ |\boldsymbol{\tau}(\boldsymbol{u})| \leq \tau_{0} & \text { if }|\boldsymbol{D}(\boldsymbol{u})|=0,\end{array}$

or equivalently

$\boldsymbol{D}(\boldsymbol{u})= \begin{cases}\left(1-\frac{\tau_{0}}{|\tau(\boldsymbol{u})|}\right) \frac{\tau(\boldsymbol{u})}{2 \eta} & \text { if }|\boldsymbol{\tau}(\boldsymbol{u})|>\tau_{0}, \\ 0 & \text { otherwise, }\end{cases}$

where $\tau_{0}$ is the yield stress, $\eta$ is the plastic viscosity, $\boldsymbol{D}(\boldsymbol{u})=(\boldsymbol{\nabla} \boldsymbol{u}+$ $\left.\nabla \boldsymbol{u}^{T}\right) / 2$ is the rate of deformation tensor, and, for any tensor $\zeta=\left(\zeta_{i j}\right)$, the notation $|\zeta|$ represents the following matrix norm

$|\zeta|^{2}=\frac{1}{2} \zeta: \zeta=\frac{1}{2} \sum_{i, j} \zeta_{i j}^{2}$

Notice that, for $\tau_{0}=0$, we recover the constitutive law for Newtonian viscous fluids.

The equations that govern the motion of the ith particle are the following inertialess Newton-Euler equations

$0=\boldsymbol{F}_{i}^{h}+\boldsymbol{F}_{i}^{p}$,

$0=\boldsymbol{T}_{i}^{h}+\boldsymbol{T}_{i}^{p}$,

where $\boldsymbol{F}_{i}^{p}$ and $\boldsymbol{T}_{i}^{p}$ are respectively the resultant and the moment of the contact forces acting on the $i$ th particle due to the other particle coming close to it. In the whole paper, we assume that collision between particles give rise only to normal forces, which yields that the contact torque $\boldsymbol{T}_{i}^{p}$ is zero. The detail of the contact model is presented in Section 3.2. $\boldsymbol{F}_{i}^{h}$ and $\boldsymbol{T}_{i}^{h}$ denote respectively the resultant and the moment of the hydrodynamic forces acting on the ith particle which are calculated by

$\boldsymbol{F}_{i}^{h}=\int_{\partial P_{i}(t)} \boldsymbol{\sigma} \cdot \boldsymbol{n} \mathrm{d} s$

$\boldsymbol{T}_{i}^{h}=\int_{\partial P_{i}(t)}\left(\boldsymbol{x}-\boldsymbol{X}_{i}\right) \times(\boldsymbol{\sigma} \cdot \boldsymbol{n}) \mathrm{d} s$,

where $\boldsymbol{X}_{i}$ is the position of the center of the $i$ th particle and $\boldsymbol{n}$ is the unit normal vector to $\partial P_{i}(t)$ pointing out of the particle. All the moments are computed about the center of the particles. The position $\boldsymbol{X}_{i}$ of the $i$ thparticle and its angular rotation $\boldsymbol{\Theta}_{i}$ are obtained by integration of the kinematic equations

$\frac{\mathrm{d} \boldsymbol{X}_{i}}{\mathrm{~d} t}=\boldsymbol{U}_{i}, \quad \boldsymbol{X}_{i}(t=0)=\boldsymbol{X}_{i, 0}$,

$\frac{\mathrm{d} \boldsymbol{\Theta}_{i}}{\mathrm{~d} t}=\boldsymbol{\omega}_{i}, \quad \boldsymbol{\Theta}_{i}(t=0)=\boldsymbol{\Theta}_{i, 0}$.

where $U_{i}$ and $\omega_{i}$ are respectively the translational velocity and the angular velocity of the $i$ th particle.

For circular particles, Eq. (11) is completely decoupled from the other equations and may be ignored. Finally, the following boundary conditions are needed to close the system 


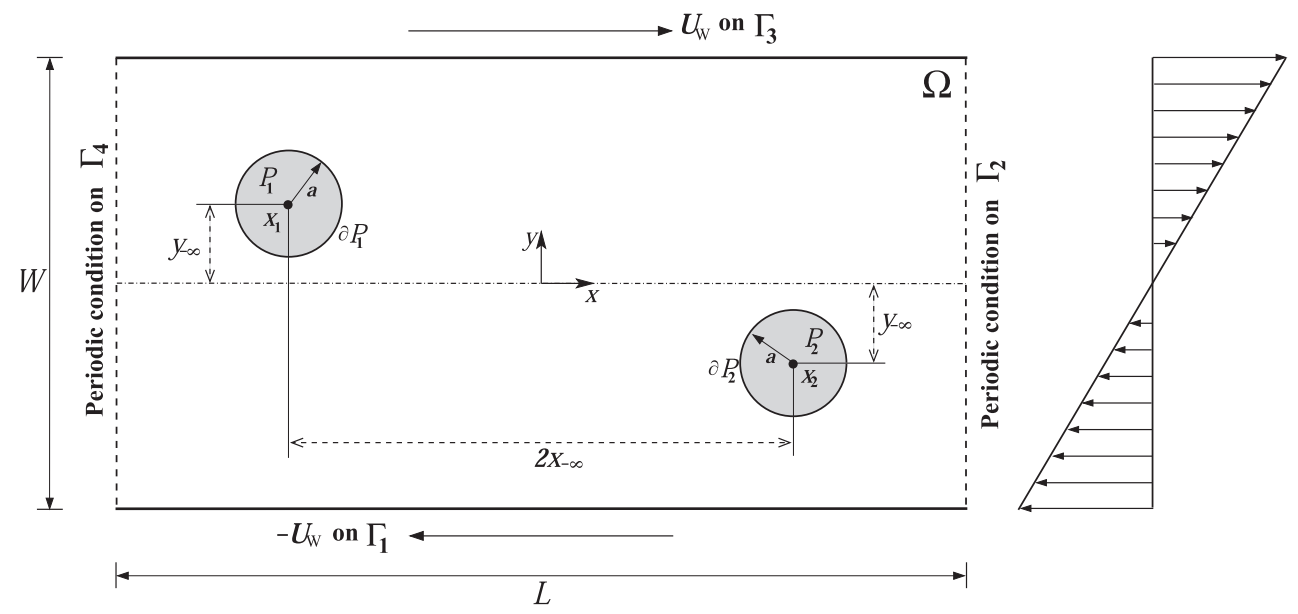

Fig. 1. Schematic description of the two-dimensional fluid-particle system: two particles $P_{1}(t)$ and $P_{2}(t)$ are suspended in a rectangular fluid domain, $\Omega$, with boundary conditions enforcing a linear shear flow far from the particles.

$\boldsymbol{u}=\boldsymbol{U}_{i}+\boldsymbol{\omega}_{i} \times\left(\boldsymbol{x}-\boldsymbol{X}_{i}\right)$ on $\partial P_{i}(t)$

$\boldsymbol{u}=\boldsymbol{u}_{\Gamma}=\dot{\gamma} y \boldsymbol{e}_{x}$ on $\Gamma_{1} \cup \Gamma_{3}$

$\boldsymbol{u}(-L / 2, y)=\boldsymbol{u}(L / 2, y) \quad \forall y \in[-W / 2, W / 2]$

Eq. (12) is the no-slip boundary condition on the particle surface. Equation (13) gives the shear flow condition on the external fluid boundaries whereas Eq. (14) states periodic conditions on $\Gamma_{2}$ and $\Gamma_{4}$. Note that, the boundary data $\boldsymbol{u}_{\Gamma}$ satisfy the compatibility condition $\int_{\Gamma} \boldsymbol{u}_{\Gamma} \cdot \boldsymbol{n} \mathrm{d} \Gamma=0$. Since inertia is neglected, no initial condition is required for the velocity field of the fluid as well as the particles. In our simulations, we use a stress-free state, $\left.\sigma\right|_{t=0}=0$ and a pressure-free state, $\left.p\right|_{t=0}=0$, as initial conditions over the whole domain.

\section{Numerical model}

\subsection{Combined weak formulation}

We use a Lagrange multiplier based fictitious domain method for solving the problem which was developed by Glowinski et al. [26-29]. The key idea of this method is to artificially fill the interior of the particles with the fluid and to introduce a Lagrange multiplier on the particle boundaries to enforce the rigid-body motion constraint. Its main advantage is that the problem, in the whole domain, can be discretized on a fixed uniform finite element grid, independent of the actual boundary of moving particles. This avoids the construction of boundary-fitted meshes for each different position of the moving particles and allows the use of fast and efficient solvers.

In this work, we follow the approach of Glowinski et al. [26] and Patankar et al. [48] in the derivation of the weak form where the fluidparticle motion is treated implicitly via a combined weak formulation in which the hydrodynamic force and torque on a particle boundary exactly cancel. The fluid flow equations are enforced inside, as well as outside, the particle boundaries. The flow inside and on each particle boundary is constrained to be a rigid-body motion using a distributed Lagrange multiplier. This multiplier can be viewed as a pseudo-force to maintain the rigid-body motion inside the particle boundary, and is analogous to the pressure in incompressible fluid flow, whose gradient is the force required to maintain the constraint of incompressibility. In the method of Glowinski et al. [26] the constraint of rigid body motion is written in the following form

$u(x)=U_{i}+\omega_{i} \times\left(x-X_{i}\right), \quad \forall x \in P_{i}$.
Thus, the translational and angular velocities of the particles appear, in some sense, as "extra unknowns", in addition to the velocity field inside the particles. Hence, the resulting algorithm requires extra conditions on the space of the distributed Lagrange multipliers if the particles are neutrally buoyant. We prefer to adopt the approach of Patankar et al. [48] in which the rate of deformation tensor is constrained to be zero inside each particle in order to impose the rigid body condition

$\boldsymbol{D}(\boldsymbol{u})=0$ in $P_{i}(i=1,2)$.

Eqs. (15) and (16) are equivalent. As a consequence, the extra unknowns are eliminated from the coupled system of equations and the resulting algorithm does not require extra conditions for handling neutrally buoyant particles.

We define the solution spaces for the velocity and pressure as

$\mathcal{V}_{u_{\Gamma}}=\left\{\boldsymbol{v} \mid \boldsymbol{v} \in\left[H^{1}(\Omega)\right]^{2}, \boldsymbol{v}=\boldsymbol{u}_{\Gamma}\right.$ on $\Gamma_{1} \cup \Gamma_{3}, \boldsymbol{v}$ periodic on $\left.\Gamma \backslash\left(\Gamma_{1} \cup \Gamma_{3}\right)\right\}$,

$\mathcal{V}_{0}=\left\{\boldsymbol{v} \mid \boldsymbol{v} \in\left[H^{1}(\Omega)\right]^{2}, \boldsymbol{v}=0\right.$ on $\left.\Gamma\right\}$,

$\mathcal{Q}=L^{2}(\Omega)$,

$\mathcal{Q}_{0}=\left\{q \mid q \in L^{2}(\Omega), \int_{\Omega} q \mathrm{~d} \Omega=0\right\}$.

The combined weak formulation can be stated as follows: For a.e. $t>0$, find $u \in \mathcal{V}_{u_{\Gamma}}, p \in \mathcal{Q}_{0}$ and $\lambda \in \Lambda(t)=\left[L^{2}(P(t))\right]^{2 \times 2}$ such that

$$
\begin{aligned}
& \int_{\Omega} \tau(\boldsymbol{u}): \boldsymbol{D}(\boldsymbol{v}) \mathrm{d} \Omega-\int_{\Omega} p \boldsymbol{\nabla} \cdot \boldsymbol{v} \mathrm{d} \Omega+\int_{P(t)} \lambda: \boldsymbol{D}(\boldsymbol{v}) \mathrm{d} \Omega=\int_{\Omega} \boldsymbol{A}_{c} \cdot \boldsymbol{v} \mathrm{d} \Omega, \\
& \forall \boldsymbol{v} \in \mathcal{V}_{0},
\end{aligned}
$$

$\int_{\Omega} q \boldsymbol{\nabla} \cdot \boldsymbol{u} \mathrm{d} \Omega=0, \quad \forall q \in \mathcal{Q}$

$\int_{P(t)} \boldsymbol{D}(\boldsymbol{u}): \lambda \mathrm{d} \Omega=0, \quad \forall \lambda \in \Lambda(t)$.

where $\lambda(t)$ is the distributed Lagrange multiplier for the rigidity constraint and $\boldsymbol{A}_{c}$ is a continuous force field defined on the whole domain which accounts for all the forces applied to the ith particle due to collision

$\boldsymbol{A}_{c}(\boldsymbol{x})=\left\{\begin{array}{ll}\boldsymbol{A}_{c, i} & \text { if } x \in P_{i} \\ 0 & \text { otherwise }\end{array}\right.$ with $\boldsymbol{A}_{c, i}=\frac{1}{V_{i}} \boldsymbol{F}_{i}^{p}$

where $V_{i}=\pi a^{2}$ is the surface of the ith particle. Putting (20) into the right hand side of (17) for a velocity field complying with the constraint 


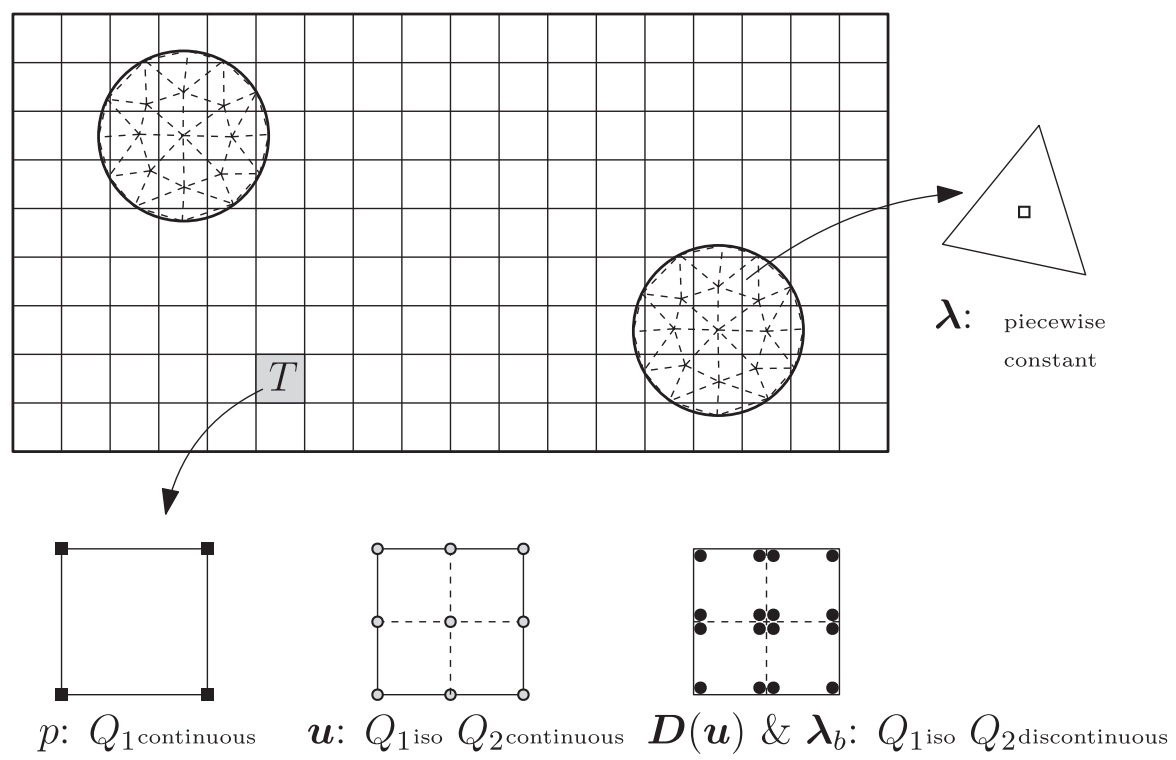

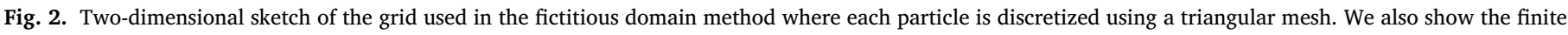
element approximations for the pressure $p$, velocity $\boldsymbol{u}$, deformation-rate tensor $\boldsymbol{D}(\boldsymbol{u})$ and Lagrange multipliers $\lambda$ and $\lambda_{b}$.

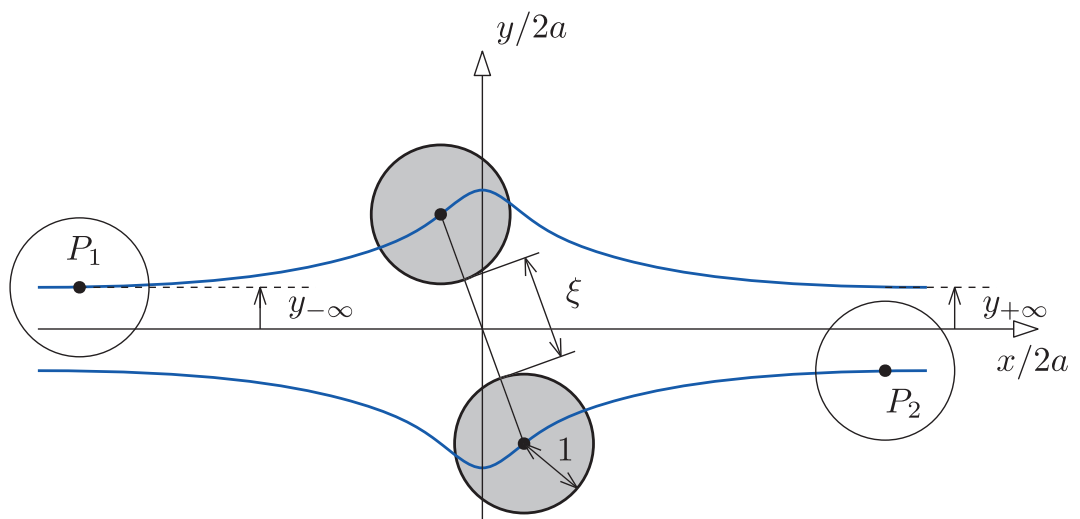

Plane of symmetry $x=0$

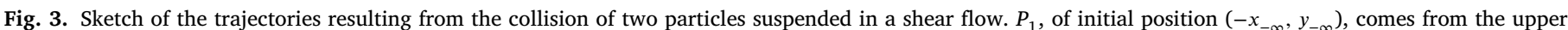

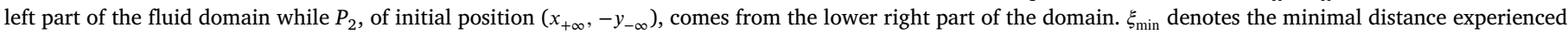
by the particles during their collision. $y_{+\infty}$ (resp. $-y_{+\infty}$ ) denotes the final vertical position of $P_{1}$ (resp. $P_{2}$ ).

of rigid body (15) or (16) for each particle yields the power of the collision forces. The above formulation has two important advantages. The first is that the hydrodynamic force and torque do not need to be computed. The second advantage is that the resulting finite element schemes are not subject to a numerical instability which can arise when the equations of fluid and particle motion are integrated as a coupled system with explicitly computed force and torque [see 33].

The numerical implementation of the formulation (17)-(19) is difficult because of the non-differentiable form of $\tau(\boldsymbol{u})$ at the yield point and the indeterminate nature of the stress in the unyielded regions [61]. Two main approaches have been developed to overcome the computational difficulties associated with the discontinuity of the constitutive law, namely the regularization techniques [47] and the multiplier method $[17,19]$. In the regularization methods the nonsmooth equation is replaced by an approximated one in which the material at the rigid region behaves like a very viscous fluid, whereas the multiplier method is equivalent to the original constitutive law. The work of Frigaard and Nouar [24] compared both methods and showed that the regularization method failed in accurately tracking the yield surfaces.
Following the multiplier method of Dean et al. [18], the constitutive equation (4), is rewritten as follows

$\boldsymbol{\tau}(\boldsymbol{u})=2 \eta \boldsymbol{D}(\boldsymbol{u})+\tau_{0} \lambda_{b}$,

where $\lambda_{b}$ is a tensor-valued function (the multiplier) verifying

$\lambda_{b}=\lambda_{b}^{T}, \quad\left|\lambda_{b}\right| \leq 1, \quad \lambda_{b}: D(u)=|\boldsymbol{D}(u)|$.

The above relations are equivalent to

$\lambda_{b}(t)=\mathcal{P}_{\Lambda_{b}}\left[\lambda_{b}(t)+\alpha \boldsymbol{D}(\boldsymbol{u}(t))\right], \forall \alpha>0$, a.e. on $(0, T)$

where $\Lambda_{b}$ is the closed convex set of $\left[L^{2}(\Omega)\right]^{2 \times 2}$ defined by

$\Lambda_{b}=\left\{\zeta\left|\zeta \in\left[L^{2}(\Omega)\right]^{2 \times 2}, \zeta=\zeta^{T},\right| \zeta(x) \mid \leq 1\right.$, a.e. on $\left.\Omega\right\}$,

and $\mathcal{P}_{\Lambda_{b}}$ is the orthogonal-projection operator from $\left[L^{2}(\Omega)\right]^{2 \times 2}$ onto $\Lambda_{b}$; it verifies

$\mathcal{P}_{\Lambda_{b}}(\zeta)(x)=\zeta(x)[\max (1,|\zeta(x)|)]^{-1}$, a.e. on $\Omega, \forall \zeta \in\left[L^{2}(\Omega)\right]^{2 \times 2}$.

Substituting (21) into (17) yields 


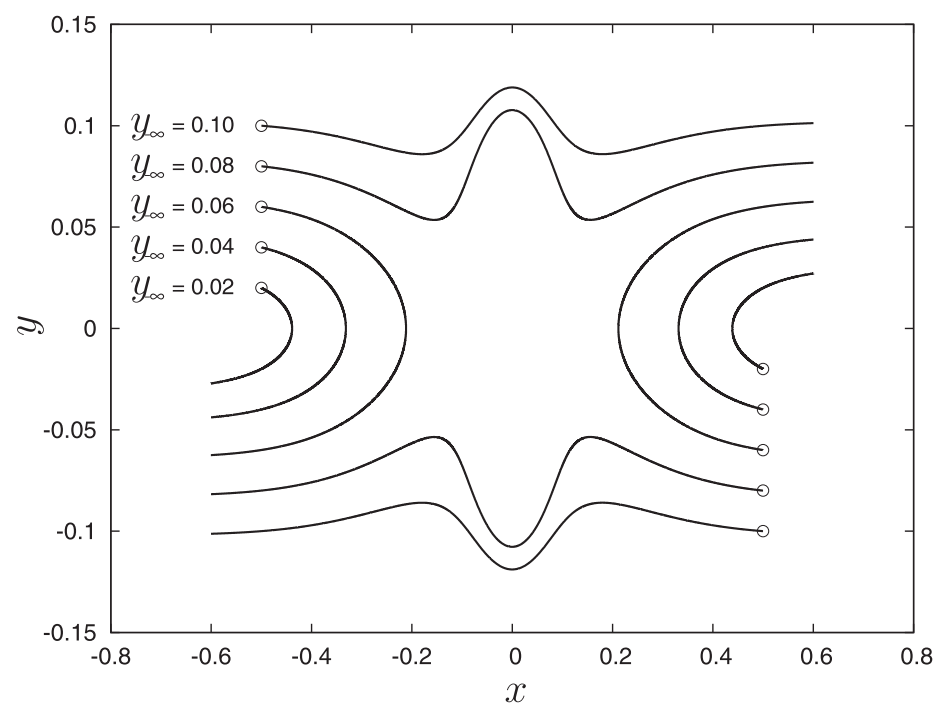

Fig. 4. Validation: particle trajectories in a Stokes flow, with the same geometric parameters as Choi et al. [9] $\left(a=0.1, x_{\infty}=0.5, L=4, W=1\right)$. For the sake of comparison, data are not plotted here in the dimensionless space, as in Choi et al. [9, Figure 31].

$\int_{\Omega}\left(2 \eta \boldsymbol{D}(\boldsymbol{u})+\tau_{0} \lambda_{b}\right): \boldsymbol{D}(\boldsymbol{v}) \mathrm{d} \Omega-\int_{\Omega} p \boldsymbol{\nabla} \cdot \boldsymbol{v} \mathrm{d} \Omega+\int_{P(t)}$

$\lambda: \boldsymbol{D}(\boldsymbol{v}) \mathrm{d} \Omega=\int_{\Omega} \boldsymbol{A}_{c} \cdot \boldsymbol{v} \mathrm{d} \Omega$,

From now, we non-dimensionalize all lengths by the radius of the particles $a$, the time by the inverse of the shear rate $\dot{\gamma}^{-1}$, the velocity by $\dot{\gamma} a$ and the pressure and total stress by $\eta \dot{\gamma}$. We also introduce the classical dimensionless Bingham number

$B n=\frac{\tau_{0}}{\eta \dot{\gamma}}$.

For the sake of simplicity we do not introduce different notations for the dimensionless quantities; Eq. (26) now reads

$$
\begin{aligned}
& \int_{\Omega}\left(2 \boldsymbol{D}(\boldsymbol{u})+B n \lambda_{b}\right): \boldsymbol{D}(\boldsymbol{v}) \mathrm{d} \Omega-\int_{\Omega} p \boldsymbol{\nabla} \cdot \boldsymbol{v} \mathrm{d} \Omega+\int_{P(t)} \lambda: \boldsymbol{D}(\boldsymbol{v}) \mathrm{d} \Omega \\
& =\int_{\Omega} \boldsymbol{A}_{\boldsymbol{c}} \cdot \boldsymbol{v} \mathrm{d} \Omega,
\end{aligned}
$$

Eq. (27), combined with the dimensionless forms of Eqs. (18), (19) and (23), constitute the complete set of the dimensionless combined weak formulation.

\subsection{Collision strategy}

For handling more than one particle in the fluid, a collision model is required to prevent particles from interpenetrating each other. Theoretically, smooth rigid particles in a Newtonian fluid cannot touch since the viscous fluid in the narrow gap between close particles exerts lubrication forces which prevent the collision within a finite time [16]. However, in numerical simulations, particles can come into contact or even overlap each other due to the discretization errors. Moreover, in experiments, particles are never perfectly smooth. It has been shown in Blanc et al. [6] that any small surface roughness causes the particles to come into contact, which impacts the suspensions microstructure and behavior. This point thus has to be finely controlled in simulations.

Different collision models have been developed to prevent interpenetration and handle the interparticle direct interaction that results from collision. The first approach consists in accurately approximating the lubrication forces by locally refining the gap zone between the particles as proposed by $\mathrm{Hu}$ [32]. Furthermore, the time step has to be reduced significantly in order to resolve the collision which makes the resulting scheme computationally expensive. To handle this problem numerically, less time consuming numerical models have been proposed, such
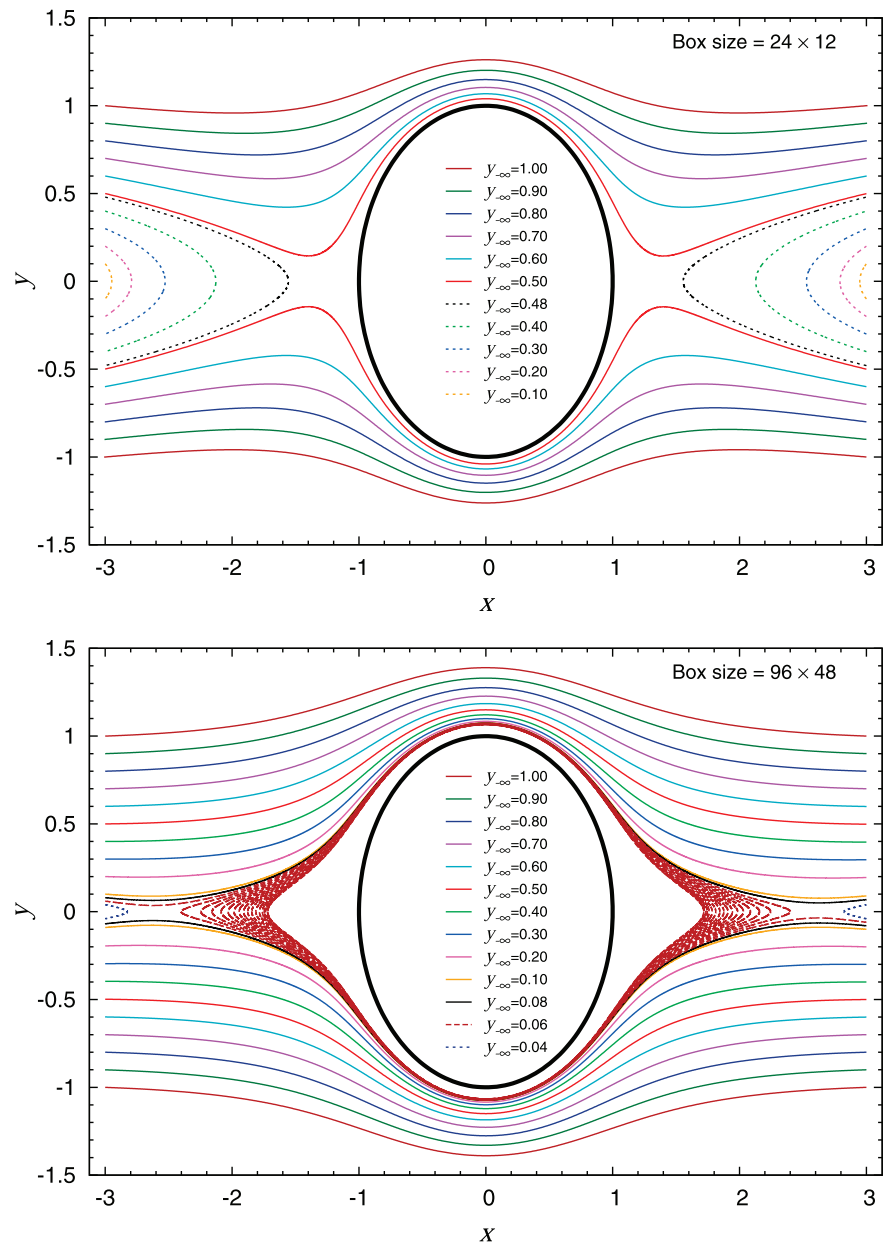

Fig. 5. Wall effect: trajectories for two different box sizes. The local minimum of the trajectories (previously observed experimentally is eliminated when using a large box. Dotted lines correspond to reversing trajectories, the dashed line is a closed trajectory. 


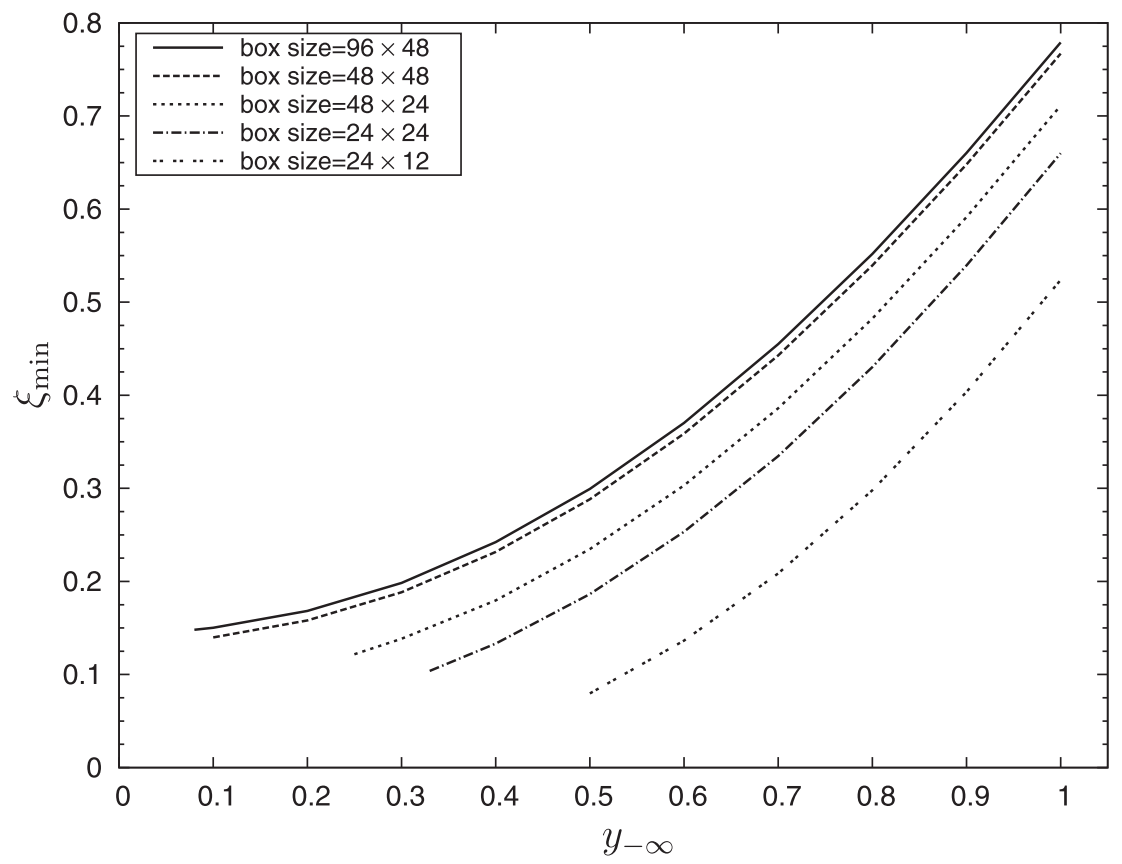

Fig. 6. Wall effect: minimum separation distance during purely hydrodynamic interactions, $\xi_{\min }$, as a function of the initial offset $y_{-\infty}$, for different box sizes, for $B n=0$.

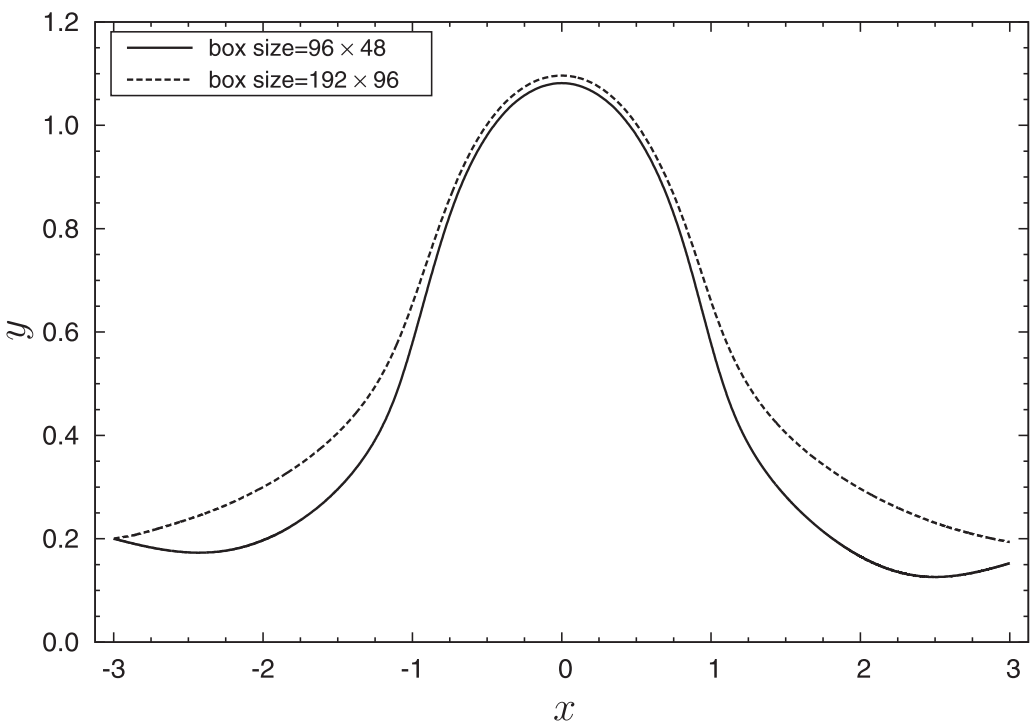

Fig. 7. Wall effect: Trajectories of particle $P_{1}$ with purely hydrodynamic interactions and two different box sizes $96 \times 48$ (solid line) and $192 \times 96$ (dashed line) for $B n=10 ; y_{-\infty}=0.2$. The local minimum is eliminated when using a large box.

as repulsive potential force models $[27,42,58]$, lubrication theory-based collision models [41,44], hard-sphere models [2,35], repulsive velocity barrier models [14], stochastic collision models [52], and adaptive collision time models [37]. In all these models, a security zone is defined around the particle such that when the gap between particles is smaller than the security zone a repelling force is activated which pushes them apart. This repelling force is usually introduced between particles to qualitatively model non-hydrodynamic effects such as surface roughness which play a major role not only in causing particle contacts but also in the rheological behavior of suspensions $[59,60]$. Therefore, the accurate numerical modeling of the collision process is crucial for the quality of the simulation and its comparison with experimental results. Some of these models have been recently analyzed and compared by Usman [56] for the simulation of particle sedimentation in two-dimensions.
Two collision models are investigated here. The first one is suggested by Glowinski et al. [27] and well-tested in the context of fictitious domain method, where a short-range repulsion force between particles that are near contact is introduced. It takes the following explicit form

$\boldsymbol{F}_{i}^{p}\left(\boldsymbol{X}_{i}\right)=\frac{1}{\delta}\left(\boldsymbol{X}_{i}-\boldsymbol{X}_{j}\right)(\varepsilon-\xi)^{2}$,

where $\delta$ is a small positive stiffness parameter that determines the magnitude of this force; $\varepsilon$ is the characteristic range of the force, $\xi=r-2$ is the dimensionless surface-to-surface separation with $r$ the dimensionless center-to-center separation. The repulsive interactions are present only for $\xi<\varepsilon$. In this approach, the choice of the stiffness parameter $\delta$ is crucial. If $\delta$ is too large, the interpenetration will not be prevented, and if it is too small, the repulsive force will be too strong and particles will bounce too much during the collision, as explained by Hu et al. [34]. In 


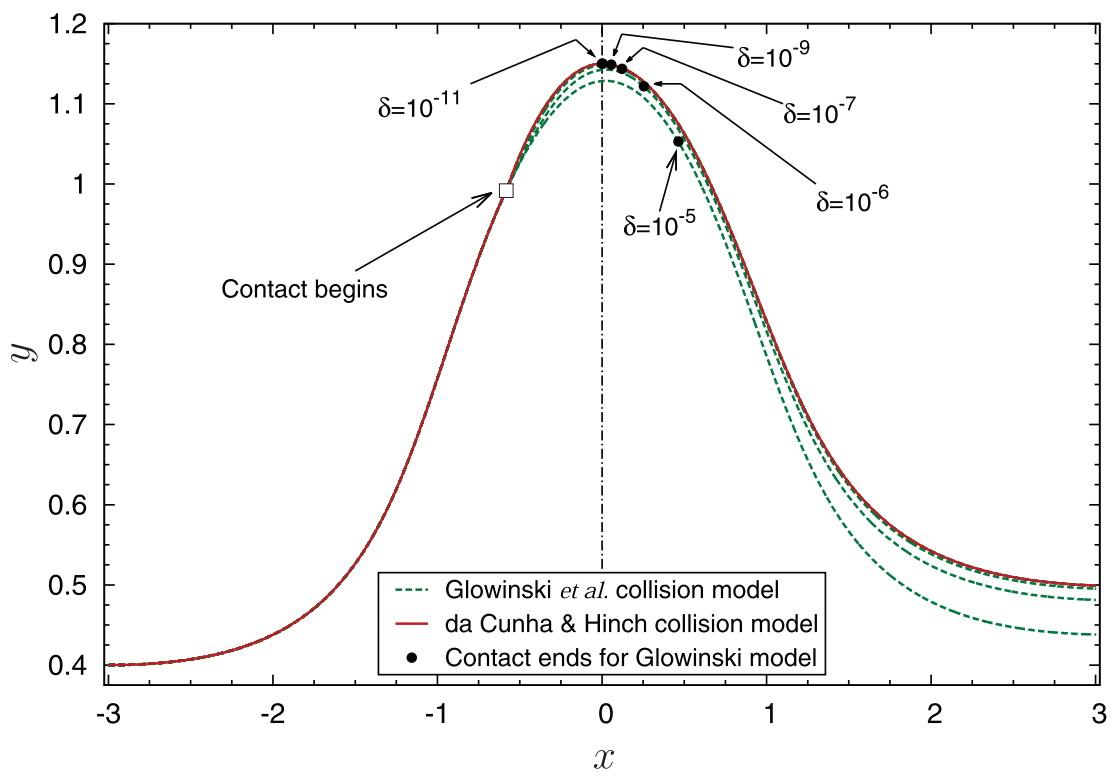

Fig. 8. Comparison between the trajectories of particle $P_{1}$ obtained with the repulsive potential force model of Glowinski et al. [27] (dashed lines) and those obtained with the surface roughness model of da Cunha and Hinch [12] (solid line) for box size $96 \times 48$. For both models the contact begins at the same point (open square). For the model of da Cunha and Hinch [12] the contact ends when the particles cross the symmetry axis $x=0$ (vertical dashed-dotted line). For the model of Glowinski et al. [27], the contact ends in the extensional quadrant depending on the value of the stiffness parameter $\delta$ (closed circles). Parameters: $B n=0, y_{-\infty}=0.4, \varepsilon=0.3$.

general, the ideal value of $\delta$ may vary from case to case and still there is no accurate theory to determine the appropriate values of this parameter. According to Glowinski et al. [26], the particle surfaces should be kept at a distance of more than one grid element apart, in order to have at most one rigid body motion constraint at each node. Anyway, in this collision scheme, there is no control on the minimum distance between the particles and the particles may still overlap.

The second collision model we use is that of da Cunha and Hinch [12] for the surface roughness. Let $\varepsilon$ be the dimensionless roughness height or the maximum allowed dimensionless film thickness between the surfaces of the two particles. Assume that all contacts between the particles have the same minimum center-to-center separation $r=2+\varepsilon$. When the particles are at their minimum separation, a normal force is exerted to prevent particles to interpenetrate but no force is exerted when the particle separate. Furthermore, the effects of the roughness is ignored up to the point of contact. The flow brings the particles together in the compressive quadrants of shear and separates them when they are in the extensional quadrants. This model changes neither the tangential motion nor the rotational motion. The model can be summarized as follows. First, we check if the surface-to-surface separation, $\xi$, is larger than the minimum separation, $\varepsilon$. If $\xi<\varepsilon$, then both particles are moved away at a distance $(\varepsilon-\xi) / 2$ along their line of centers so that the minimum constraint is always verified.

\subsection{Computational scheme}

Let $\Delta t=t^{n+1}-t^{n}>0$ be a time discretization step with $t^{n}$ the $n$th time level. For any field $f(t)$, we denote by $f^{n}$ its value at time $t=t^{n}$.

Step 1: Calculate particle velocity: given $\boldsymbol{u}^{n}$ and $P_{i}\left(t^{n}\right)$, find the translational and angular velocities of the $i$ th particle

$\boldsymbol{U}_{i}^{n}=\frac{1}{V_{i}} \int_{P_{i}\left(t^{n}\right)} \boldsymbol{u}^{n} \mathrm{~d} \Omega$

$\boldsymbol{\omega}_{i}^{n}=\frac{1}{I_{i}} \int_{P_{i}\left(t^{n}\right)}\left(x-X_{i}^{n}\right) \times u^{n} \mathrm{~d} \Omega$

where $I_{i}=\pi a^{4} / 2$ is the second moment of area of the $i$ th particle. For circular particles, the calculation of the angular velocity is not necessary.
Step 2: Update the particle positions. When particles come near contact, the time step should be reduced. We adopt here a subcycling technique similar to that considered in Patankar et al. [48]. Let $\Delta t_{k}=\Delta t / K_{t}\left(k=1, \ldots, K_{t}\right)$ be the local time step with $K_{t}$ the number of substep calculations. Set $\boldsymbol{X}_{i}^{n+1,0}=\boldsymbol{X}_{i}^{n}$. First, the new interim configuration is obtained by integrating the kinematic Eq. (10) using the explicit second-order Adams-Bashforth scheme

$\boldsymbol{X}_{i}^{* n+1, k}=\boldsymbol{X}_{i}^{n+1, k-1}+\left(\frac{3 \boldsymbol{U}_{i}^{n}-\boldsymbol{U}_{i}^{n-1}}{2}\right) \Delta t_{k}$

The first time step of the simulation is performed with an explicit firstorder Euler scheme

$\boldsymbol{X}_{i}^{* n+1, k}=\boldsymbol{X}_{i}^{n+1, k-1}+\boldsymbol{U}_{i}^{n} \Delta t_{k}$.

For nonhydrodynamic interactions, i.e. $\boldsymbol{F}_{i}^{p} \neq 0$ in (10), the particle positions in the above equations are considered as intermediate positions and should be corrected by using the collision models discussed in the previous section. For the model of Glowinski et al. [27], this correction can be seen as a second-order perturbation in time

$\boldsymbol{X}_{i}^{n+1, k}=\boldsymbol{X}_{i}^{* n+1, k}+\left[\frac{\boldsymbol{F}_{i}^{p}\left(\boldsymbol{X}_{i}^{n+1, k-1}\right)+\boldsymbol{F}_{i}^{p}\left(\boldsymbol{X}_{i}^{* n+1, k}\right)}{2 \boldsymbol{M}_{i}}\right] \frac{\Delta t_{k}^{2}}{2}$.

where $\boldsymbol{F}_{i}^{P}$ is the repulsive force given in (28), and $M_{i}$ is the pseudomass of the $i$-th particle. Note that the pseudo-mass $M_{i}$ is used only to compute the new position of the particle as a function of the contact force evaluated at time $t+\Delta t_{k}$ and is not used elsewhere in this work. Hence $M_{i}$ is just a parameter of the contact law. A careful examination of Eqs. (28) and (33) allows to show that the contact stiffness is controlled by the product $M_{i} \delta$. In the numerical computation, we choose $M_{i}=V_{i}$.

For the model of da Cunha and Hinch [12], the correction is done as follows:

$\boldsymbol{X}_{i}^{n+1, k}=\boldsymbol{X}_{i}^{* n+1, k}+\frac{\varepsilon-\boldsymbol{\xi}}{2 r}\left(\boldsymbol{X}_{i}^{* n+1, k}-\boldsymbol{X}_{j}^{* n+1, k}\right)$

This correction is equivalent to a modification of the velocity of the $i$ th particle, $\boldsymbol{U}_{i}^{n}$, by $(\varepsilon-\xi) / 2 \Delta t_{k}$ along the line of centers. Furthermore, one can verify that the minimal distance of the particles after collision is still maintained, $\left|\boldsymbol{X}_{i}^{n+1, k}-\boldsymbol{X}_{j}^{n+1, k}\right|=2+\varepsilon$. Set $\boldsymbol{X}_{i}^{n+1}=\boldsymbol{X}_{i}^{n+1, K_{t}}$, this gives the 

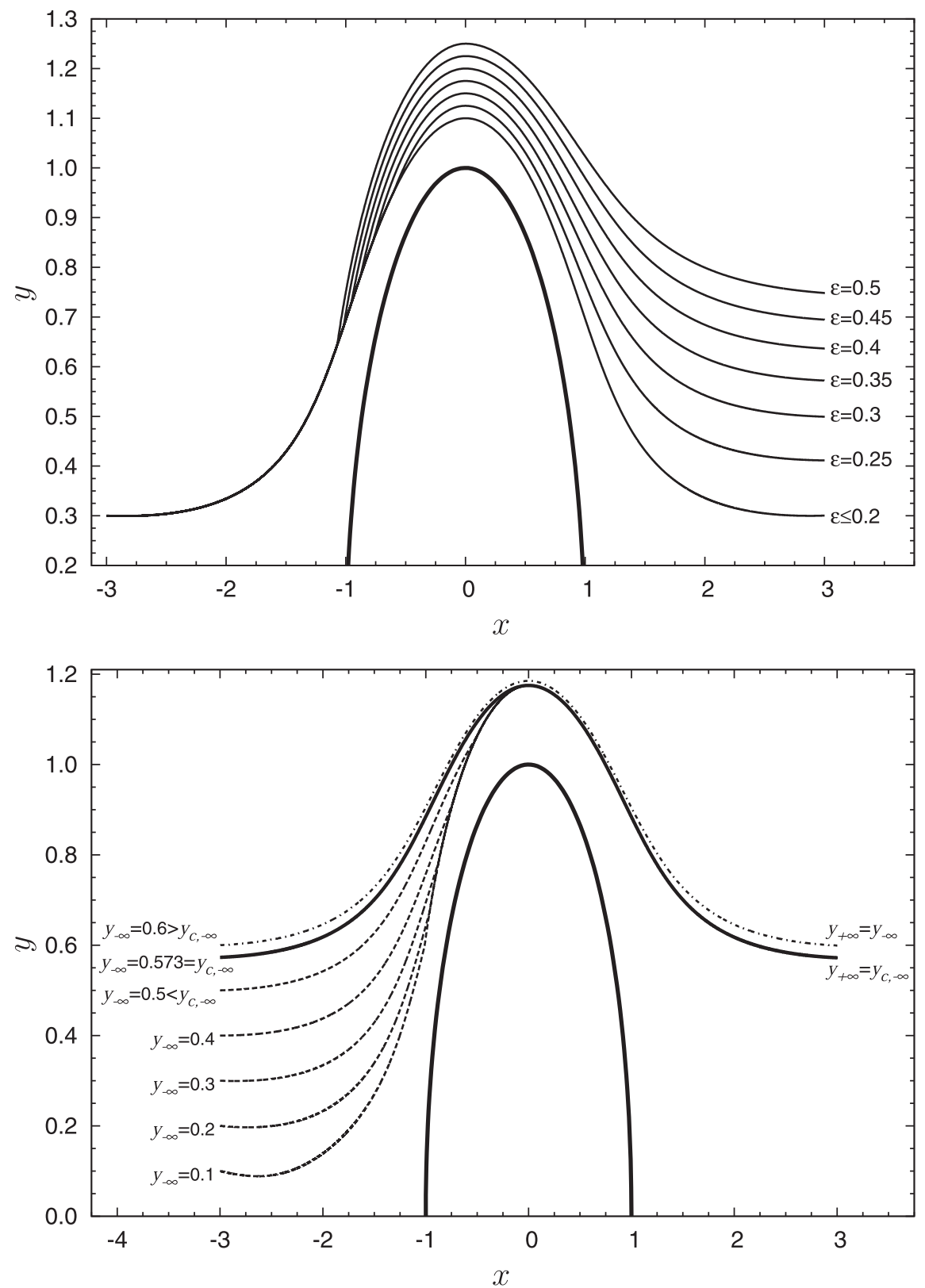

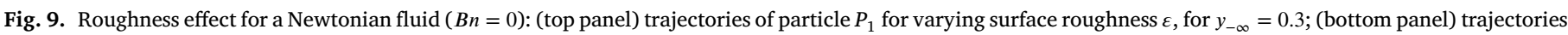
with varying initial offset $y_{-\infty}$ for surface roughness $\varepsilon=0.35$.

particle positions at time level $t^{n+1}$. In our calculations, the number of substeps is chosen as $K_{t}=1$ for $\xi \geq \varepsilon$ and $K_{t}=10$ for $\xi<\varepsilon$. Taking more than 10 substeps has no effect on the simulation.

Finally, the particle contact force $\boldsymbol{A}_{c, i}$ in (20) is given by

$\boldsymbol{A}_{c, i}^{n+1}=\frac{2}{(\Delta t)^{2}}\left(\boldsymbol{X}_{i}^{n+1}-\boldsymbol{X}_{i}^{n}-\left(\frac{\boldsymbol{U}_{i}^{n}+\boldsymbol{U}_{i}^{n-1}}{2}\right) \Delta t\right)$

This term provides an additional body force acting on the particle and is included in the combined weak formulation to be solved in the next step.

Step 3: Solving the generalized Stokes problem. Assuming all quantities at time level $n$ are known and let

$\lambda_{b}^{n+1,0}=\lambda_{b}^{n} \in \Lambda_{b}$

For $k \geq 0$, find $u^{n+1, k+1}, p^{n+1, k+1}$ and $\lambda^{n+1, k+1}$ from

$2 \int_{\Omega} \boldsymbol{D}\left(\boldsymbol{u}^{n+1, k+1}\right): \boldsymbol{D}(\boldsymbol{v}) \mathrm{d} \Omega-\int_{\Omega} p^{n+1, k+1} \boldsymbol{\nabla} \cdot \boldsymbol{v} \mathrm{d} \Omega+\int_{P\left(t^{n+1}\right)} \lambda^{n+1, k+1}:$
$\boldsymbol{D}(\boldsymbol{v}) \mathrm{d} \Omega$

$$
=\int_{\Omega} \boldsymbol{A}_{c}^{n+1} \cdot \boldsymbol{v} \mathrm{d} \Omega-B n \int_{\Omega} \lambda_{b}^{n+1, k}: \boldsymbol{D}(\boldsymbol{v}) \mathrm{d} \Omega
$$

$\int_{\Omega} q \boldsymbol{\nabla} \cdot \boldsymbol{u}^{n+1, k+1} \mathrm{~d} \Omega=0$

$\int_{P\left(t^{n+1}\right)} \boldsymbol{D}\left(\boldsymbol{u}^{n+1, k+1}\right): \lambda \mathrm{d} \Omega=0$.

Then, we calculate $\lambda_{b}^{n+1, k+1}$ from

$\lambda_{b}^{n+1, k+1}=\mathcal{P}_{\Lambda_{b}}\left[\lambda_{b}^{n+1, k}+\alpha \boldsymbol{D}\left(\boldsymbol{u}^{n+1, k+1}\right)\right]$,

where $\alpha$ is an iterative parameter and $\mathcal{P}_{\Lambda_{b}}$ is defined by (25). The above step is iterated for $k$ until the solutions are converged. The convergence of the algorithm is proved in Dean et al. [18] for the interval $0<\alpha<2 / B n$. The stopping criterion used in the numerical results 


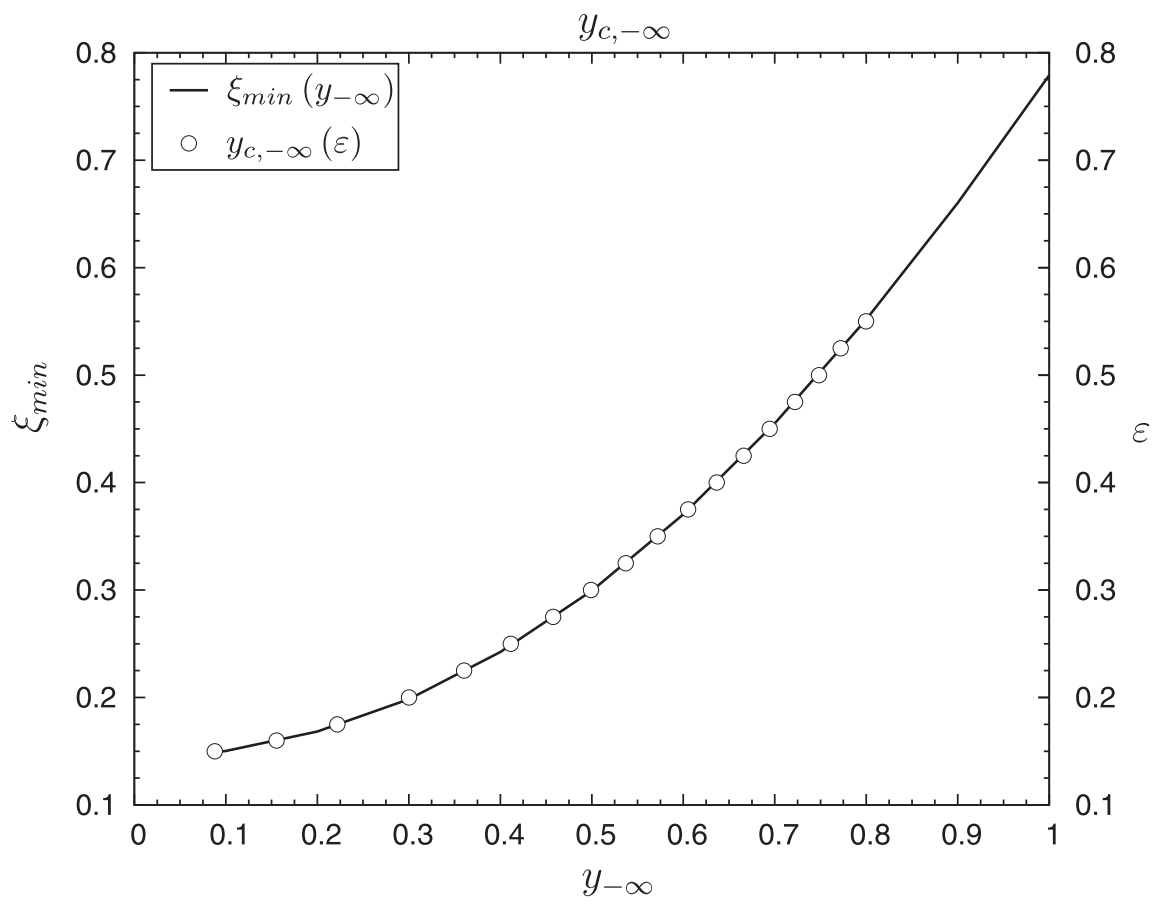

Fig. 10. Roughness effect: minimal separation distance, $\xi_{\min }$, as a function of the initial offset $y_{-\infty}$ (line), for a Newtonian fluid ( $B n=0$ ). The same curve can be used to obtain the critical offset, $y_{c,-\infty}$, as a function of the surface roughness, $\varepsilon$ (open circles). The box size is $96 \times 48$. This figure should be compared with Fig. 2 of Frechette and Drazer [23].

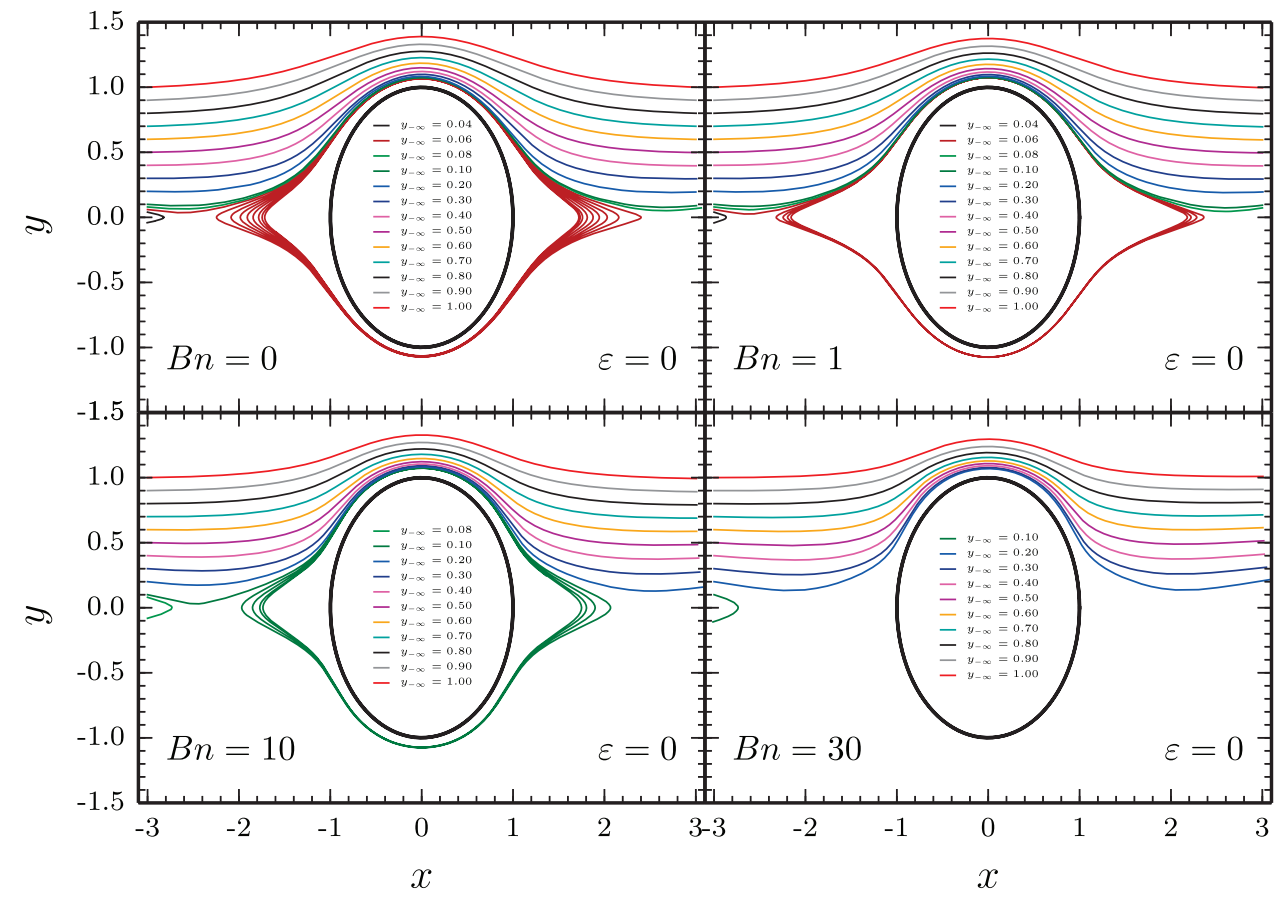

Fig. 11. Trajectories with purely hydrodynamic interactions and varying initial offset for Bingham number $B n=0,1,10,30$.

is $\left|\lambda_{b}^{n+1, k+1}-\lambda_{b}^{n+1, k}\right| \leq 10^{-4}$ for $\alpha=1 / B n$. The number of iterations required for convergence depends on the Bingham number and the initial offset. It decreases with increasing the Bingham number and increases with increasing the initial offset.

The sub-problem (35)-(37), after the discretization in space, is a finite dimensional linear problem with the following twofold saddle-point structure

$$
\left[\begin{array}{ccc}
\boldsymbol{A} & \boldsymbol{B}^{T} & \boldsymbol{C}^{T} \\
\boldsymbol{B} & 0 & 0 \\
\boldsymbol{C} & 0 & 0
\end{array}\right]\left[\begin{array}{l}
\boldsymbol{u} \\
p \\
\lambda
\end{array}\right]=\left[\begin{array}{c}
\boldsymbol{f} \\
0 \\
0
\end{array}\right]
$$

where each row corresponds to one of the equations (35)-(37). In (39), $\boldsymbol{A}$ is the Laplacian matrix for the velocity, $\boldsymbol{B}$ is the divergence matrix and $\boldsymbol{C}$ is the coupling matrix between the fluid velocity and the Lagrange multiplier. This problem can be considered as a generalized Stokes problem, 

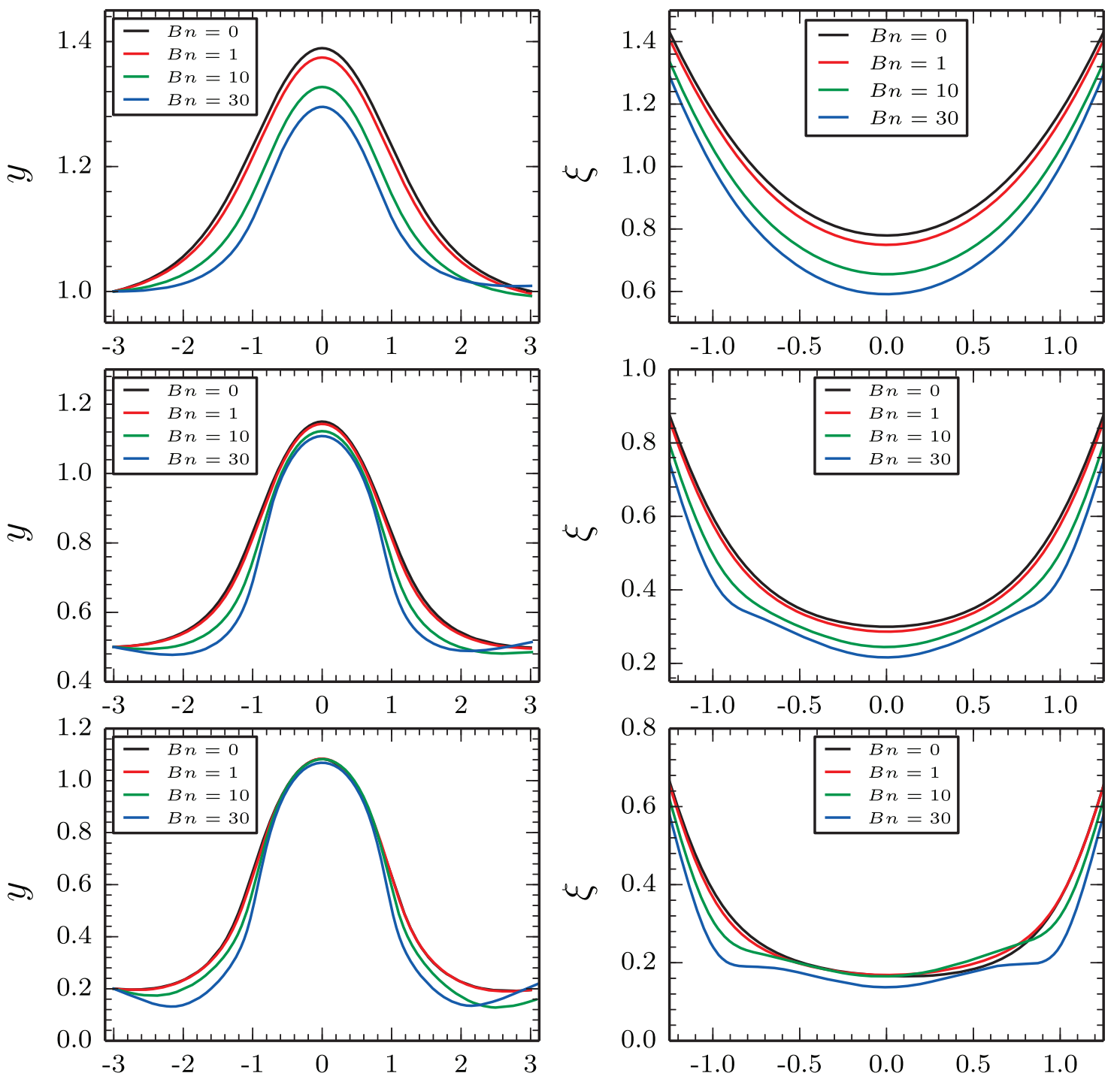

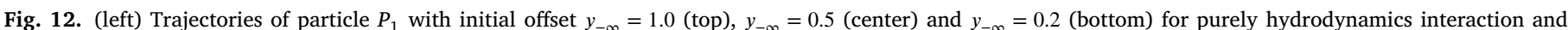

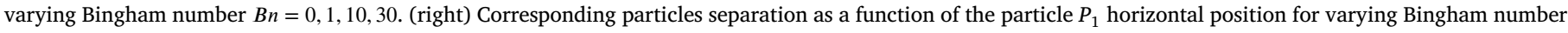
$B n=0,1,10,30$. Initial offset $y_{-\infty}=1.0$ (top), $y_{-\infty}=0.5$ (center) and $y_{-\infty}=0.2$ (bottom).

and it is solved using an Uzawa/conjugate gradient algorithm together with a fast Fourier transform based solver. The stopping criterion of the algorithm is $\left\|\boldsymbol{r}^{(k)}\right\| /\left\|\boldsymbol{r}^{(0)}\right\| \leq \varepsilon_{c g}$ where $\boldsymbol{r}^{(k)}$ is the residual error at the $k$ th iteration, and $\varepsilon_{c g}$ is a given tolerance. It typically takes less than 5 iterations in our simulations to achieve convergence when $\varepsilon_{c g}=5 \times 10^{-8}$.

The set of equations (35)-(38) are discretized in space by using the Galerkin finite-element method. We introduce a regular finite-element partition $\mathcal{T}_{h}^{\Omega}$ of $\Omega$ made of squares, where $h$ is the mesh size. Then, a twice finer partition $\mathcal{T}_{h / 2}^{\Omega}$ is obtained by joining the midpoints of the edges of $\mathcal{T}_{h}^{\Omega}$, dividing each square into 4 similar subsquares. For the discrete velocity-pressure spaces, we use continuous linear polynomials for the velocity defined on the finer grid $\mathcal{T}_{h / 2}^{\Omega}$ (the so-called $Q_{1}$-iso- $Q_{2}$ elements) and continuous linear polynomials $\left(Q_{1}\right)$ for the pressure defined on the grid $\mathcal{T}_{h}^{\Omega}$, as shown in Fig. 2. This choice of the finite dimensional spaces satisfies the Babuska-Brezzi inf-sup condition, and guarantees well-posedness of the formulation. For the discretization of the Lagrange multiplier $\lambda(t)$, we approximate the functional space $\Lambda(t)$ by piecewise constant functions defined on a triangular mesh, $\mathcal{T}_{h_{i}}^{P_{i}(t)}$, moving with each rigid body $P_{i}(t)$. A linear interpolation is used to project $\lambda$ from the particle mesh to the uniform background mesh and to project $\boldsymbol{u}$ from the background mesh to the particle mesh. Thanks to the rotation invariance property of the circular particles, the mesh $\mathcal{T}_{h_{i}}^{P_{i}(t)}$ is obtained by translating $\mathcal{T}_{h_{i}}^{P_{i}(0)}$ by the vector $\boldsymbol{X}_{i}(t)-\boldsymbol{X}_{i}(0)$. According to Glowinski et al. [27], some compatibility conditions have to be satisfied between the spaces used to approximate $\{\boldsymbol{u}, p\}$ and $\lambda$. Let $h_{\boldsymbol{u}}=h / 2$ and $h_{i}$ be the mesh sizes associated to the velocity grid $\mathcal{T}_{h / 2}^{\Omega}$ and to the rigid body mesh $\mathcal{T}_{h_{i}}^{P_{i}(t)}$. A relation of the form $h_{\boldsymbol{u}} \sim \kappa h_{i}$ with $0.1<\kappa<1$ is needed in order to satisfy some inf-sup condition, as explained in Court et al. [10]. In our numerical computations we take $h_{u}=0.56 h_{i}$ which seems to be a good compromise between these two alternatives.

One of the most important issue of the fictitious domain method is the calculation of integrals involving the distributed multiplier. Let $\mathcal{T}_{h}^{(t)}$ be the intersection between each edge of each element in $\mathcal{T}_{h_{i}}^{P_{i}(t)}$ and $\mathcal{T}_{h / 2}^{\Omega}$. The partition $\mathcal{T}_{h}^{(t)}$ contains a set of edges $S$. We approximate the last term of the left-hand side of Eq. (35) with

$\int_{P_{i}(t)} \lambda_{h}: \boldsymbol{D}_{h}\left(\boldsymbol{v}_{h}\right) \mathrm{d} \Omega=\int_{\mathcal{T}_{h_{i}}^{P_{i}(t)}} \lambda_{h}: \boldsymbol{D}_{h}\left(\boldsymbol{v}_{h}\right) \mathrm{d} \Omega=\sum_{S \in \mathcal{T}_{h}^{(t)}} \int_{S} \boldsymbol{v}_{h} \cdot \boldsymbol{n}_{S} \mathrm{~d} S$

where $\boldsymbol{n}_{S}$ is the unit outward normal to $S$. The last integral in (40) is evaluated numerically by using an appropriate quadrature rule over $S$. Since 


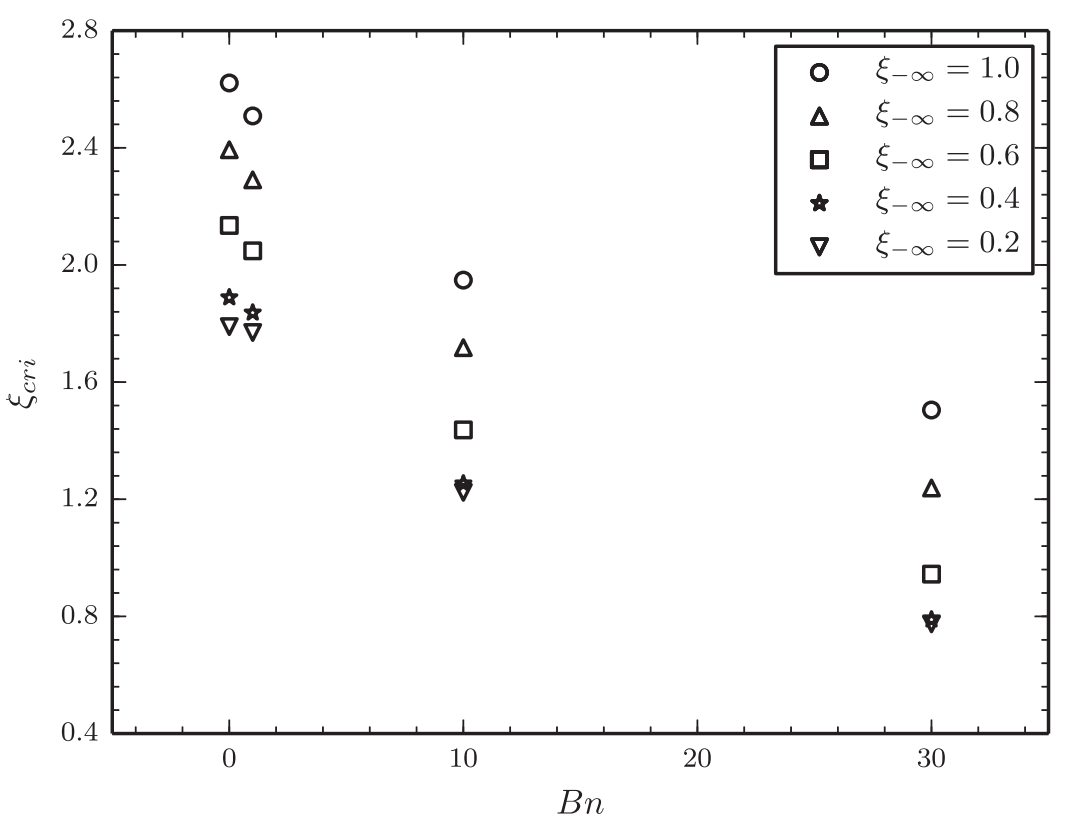

Fig. 13. Critical interparticle distance $\xi_{c r i}$ (i.e. interparticle distance of departure from the flow driven motion) as a function of the Bingham number $B n$ for several values of the initial offset $\xi_{-\infty}$.

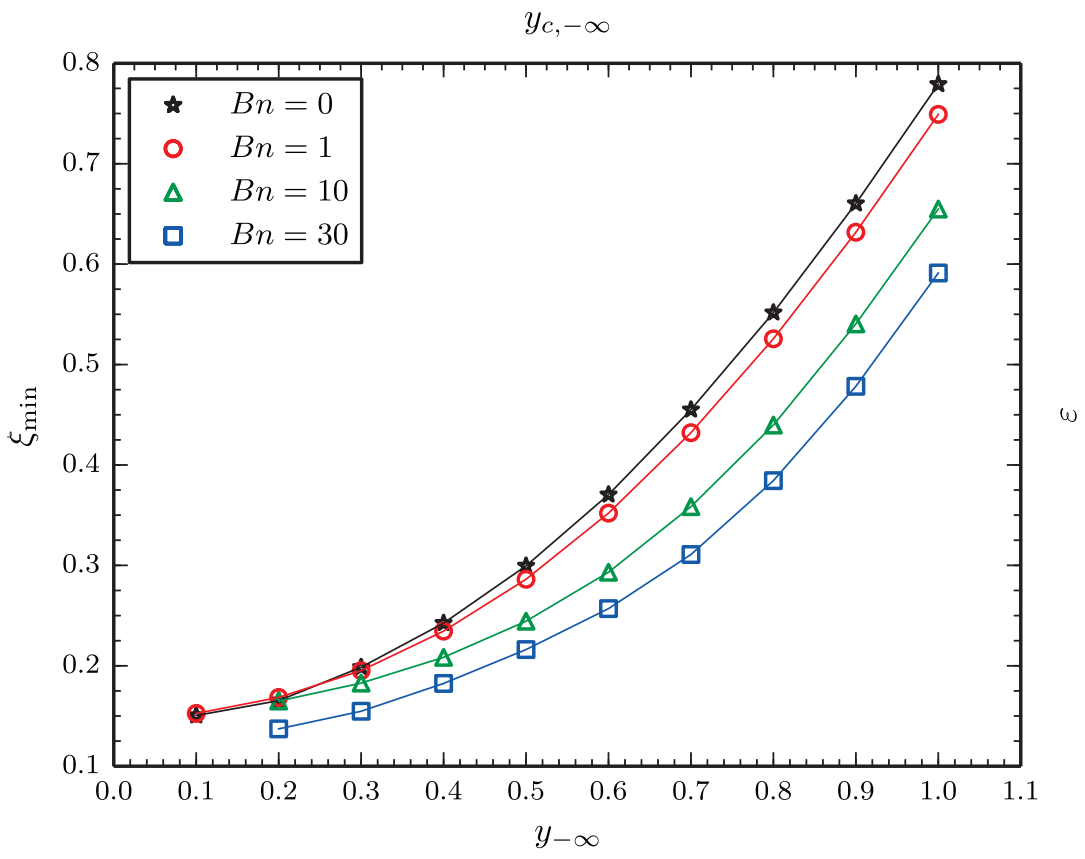

Fig. 14. Minimal separation distance, $\xi_{\min }$, as a function of the initial offset $y_{-\infty}$, for different $B n$. The same curve can be used to obtain the critical offset, $y_{c,-\infty}$, as a function of the surface roughness, $\varepsilon$ for each $B n$.

the finite element space used for the velocity includes polynomials up to degree one in each direction, the integrand in (40) involves polynomials up to degree 2. In our implementation we use a 3-point Newton-Cotes rule which is exact for polynomials of degree 2 over segments.

\subsection{Computation times}

Computations have been realized on a linux workstation (64 Go Memory, xeon E5-2650@2.00 HHz). The code has not been parallelized. As regards the computing time, for a $96 \times 48$ box, the full computation of one pair particle trajectory in the Binghamian regime $(B n \neq 0)$ requires about $20 \mathrm{~h}$.

\section{Results}

\subsection{Sketch of a trajectory and definitions}

In Fig. 3, we show a sketch of the particle trajectories.

The initial positions of the particles are $\left(-x_{-\infty}, y_{-\infty}\right)$ and $\left(x_{-\infty}\right.$, $\left.-y_{-\infty}\right)$. Their minimum separation distance during collision is denoted $\xi_{\min }$. Their final positions are $\left(x_{+\infty}, y_{+\infty}\right)$ and $\left(-x_{+\infty},-y_{+\infty}\right)$. For convenience, the final positions are set as $x_{+\infty}=x_{-\infty}$. For particles interacting only through hydrodynamic interaction, symmetric trajectories are expected (ie $y_{+\infty}=y_{-\infty}$ ) as a natural consequence of time reversibility. 

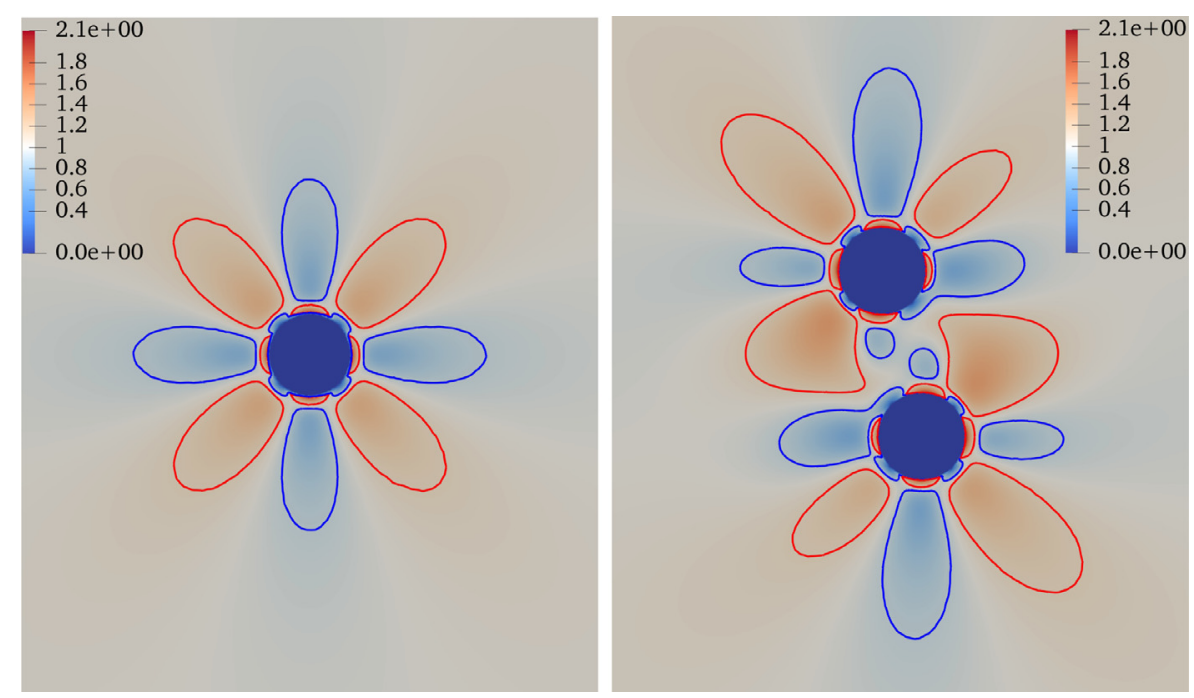

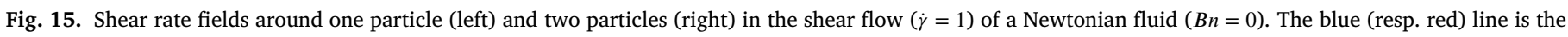
$\dot{\gamma}=0.9$ (resp. $\dot{\gamma}=1.1$ ) isoline. (For interpretation of the references to colour in this figure legend, the reader is referred to the web version of this article.)
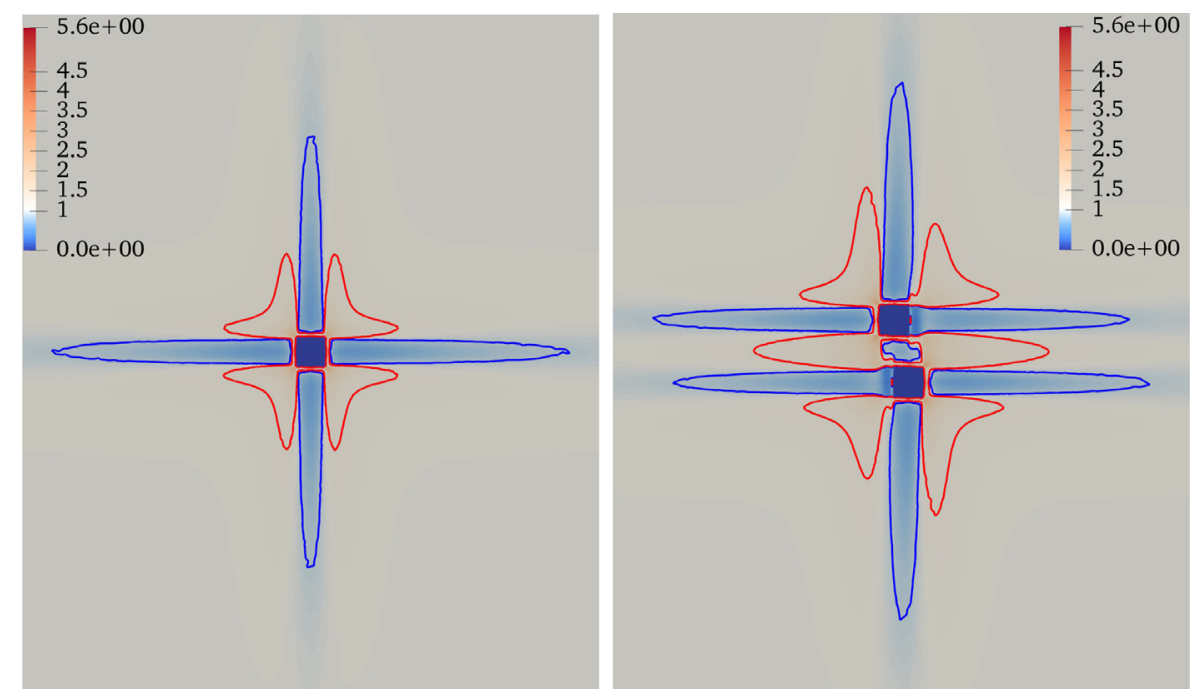

Fig. 16. Shear rate fields around one particle (left) and two particles (right) in the shear flow $(\dot{\gamma}=1)$ of a Bingham fluid $(B n=30)$. The blue (resp. red) line is the $\dot{\gamma}=0.9$ (resp. $\dot{\gamma}=1.1$ ) isoline. (For interpretation of the references to colour in this figure legend, the reader is referred to the web version of this article.)

In the following, all the numerical simulation were performed for $x_{-\infty}=-3$.

\subsection{Validation}

In order to validate our code, we first consider pair trajectories of 2D particles in an inertialess Newtonian fluid, with the same geometric parameters as Choi et al. [9], in the absence of contact forces. The trajectories are depicted in Fig. 4. The agreement with figure 31 in Choi et al. [9] is excellent.

\subsection{Box size effects}

In this subsection, we evaluate the impact of working with a finite size box on the results. In Fig. 5 we show the pair trajectories in a Newtonian fluid $(B n=0)$, for 2 different box sizes.

When the box is too small, the particles tend to first move downwards. There are reverse trajectories (dotted lines in Fig. 5) which tend to replace the closed trajectories (dashed lines), due to hydrodynamic interactions between the particles and the walls. The local minimum of the trajectories (previously observed experimentally (e.g., Snijkers et al. [51, Figure 7]) and numerically (e.g., Choi et al. [9, Figure 31]) is thus eliminated when using a large box.

The box size also has an impact on the minimum separation distance during collision $\xi_{\min }$. The values of $\xi_{\min }$ as a function of the initial vertical position $y_{-\infty}$ are depicted in Fig. 6 for different box sizes.

$\xi_{\min }$ is an increasing function of $y_{-\infty}$ in all cases. However, for small boxes, no data are obtained below a critical value of $y_{-\infty}$ because of the reverse trajectories. Moreover, at a given $y_{-\infty}, \xi_{\min }$ is much lower for small boxes. It is readily seen in Fig. 6 that $\xi_{\text {min }}$ converges (to the value for an infinite domain) when the box size is increased. A box size equal to $96 \times 48$ then appears to be a good compromise between precision and computation cost.

In a Binghamian fluid, the influence of the box walls is further enhanced at large Bingham number by plastic effects, as illustrated in Fig. 7 for a Bingham number $B n=10$.

The trajectory for a $96 \times 48$ box can be compared to the Newtonian case $(B n=0)$ of Fig. 5 . It is seen that for $B n=10$, wall effects are still present, leading the particle to first move downwards, by contrast with the case $B n=0$. This effect is eliminated by increasing the box 


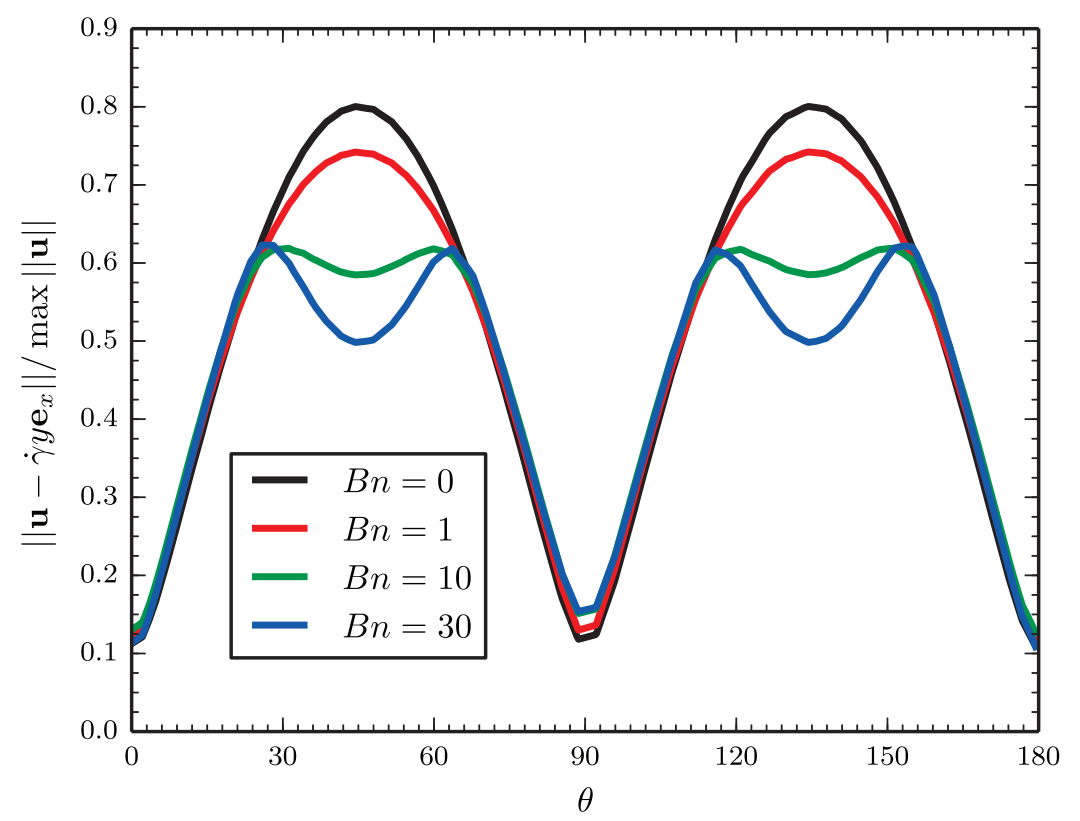

Fig. 17. Norm of the disturbance velocity around one particle at fixed distance from the particle center $(r / a=2)$ for several Bingham number.

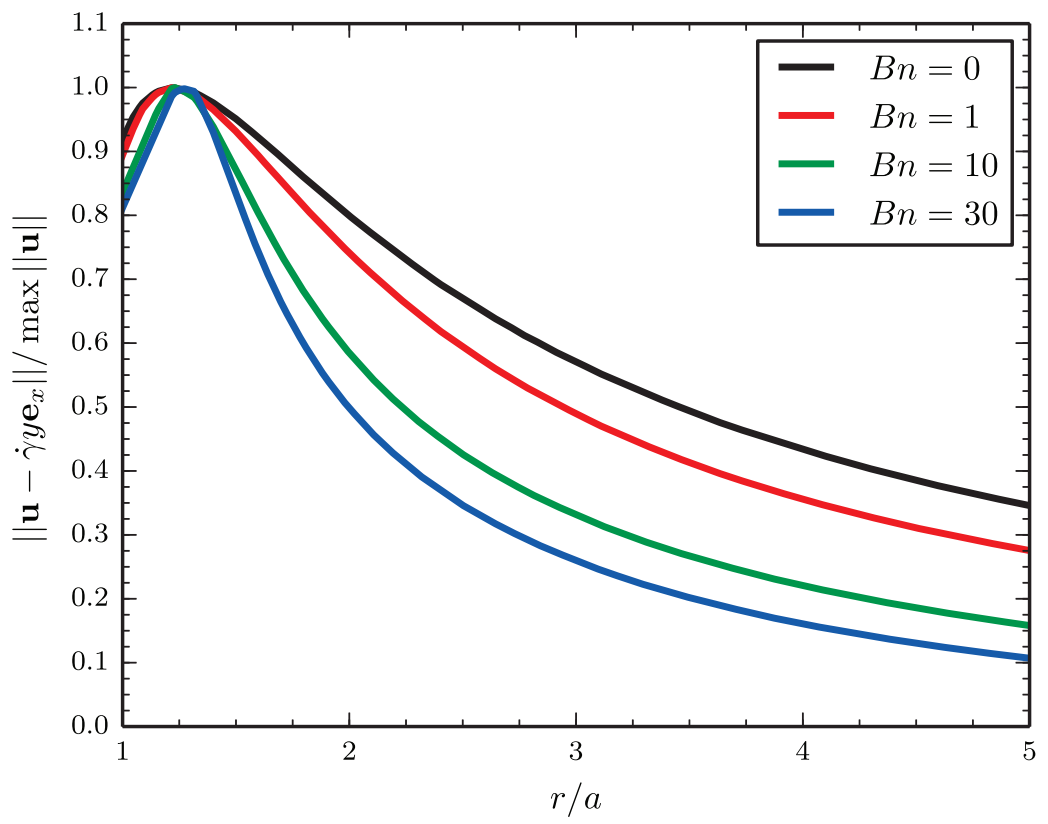

Fig. 18. Norm of the disturbance velocity around one particle along the $\theta=45^{\circ}$ direction for several Bingham numbers.

size again. However, the computational cost is too expensive at such box sizes. In the following we will thus mainly present results obtained using boxes with size $96 \times 48$. It is readily seen in Figs. 7, 11, 12 and 19 that such a box size does not allow to eliminate all the hydrodynamical interactions between the particles and the walls for the largest Bingham number values and the smaller initial offsets. As we are mainly interested in this work to the effect of short range hydrodynamical interparticle interactions and surface roughness on collision-induced vertical displacement (ie difference between the final offset and the initial offset $\left.y_{+\infty}-y_{-\infty}\right)$ it is believed that this effect is weak enough not to affect the observed trends and it remains possible to study the influence of the initial offset $y_{-\infty}$, the roughness $\varepsilon$ and the Bingham number $B n$ on this process. Furthermore the departure from symmetry observed in Fig. 12 (left) provides an estimate of the numerical error in our sim- ulations, which is increased as $B n$ is increased: we can not go beyond $B n=30$ in a reasonable computation time.

\subsection{Surface roughness effects}

In Fig. 8 we compare the trajectories obtained for the two models of contact studied in this paper, in the case of a Newtonian fluid $(B n=0)$.

It is clearly observed that both models are equivalent when the stiffness parameter $\delta$ of the Glowinski et al. [27] model (Eq. (28)) approaches zero, i.e., in the limit of infinite stiffness. This result raises doubt about the $\mathrm{Hu}$ et al. [33] claim that small values for $\delta$ are not physically relevant. As Hu et al. [33] have provided no quantitative arguments to support their opinion we do not further discuss this point in the sequel. Furthermore the da Cunha and Hinch [12] model being 


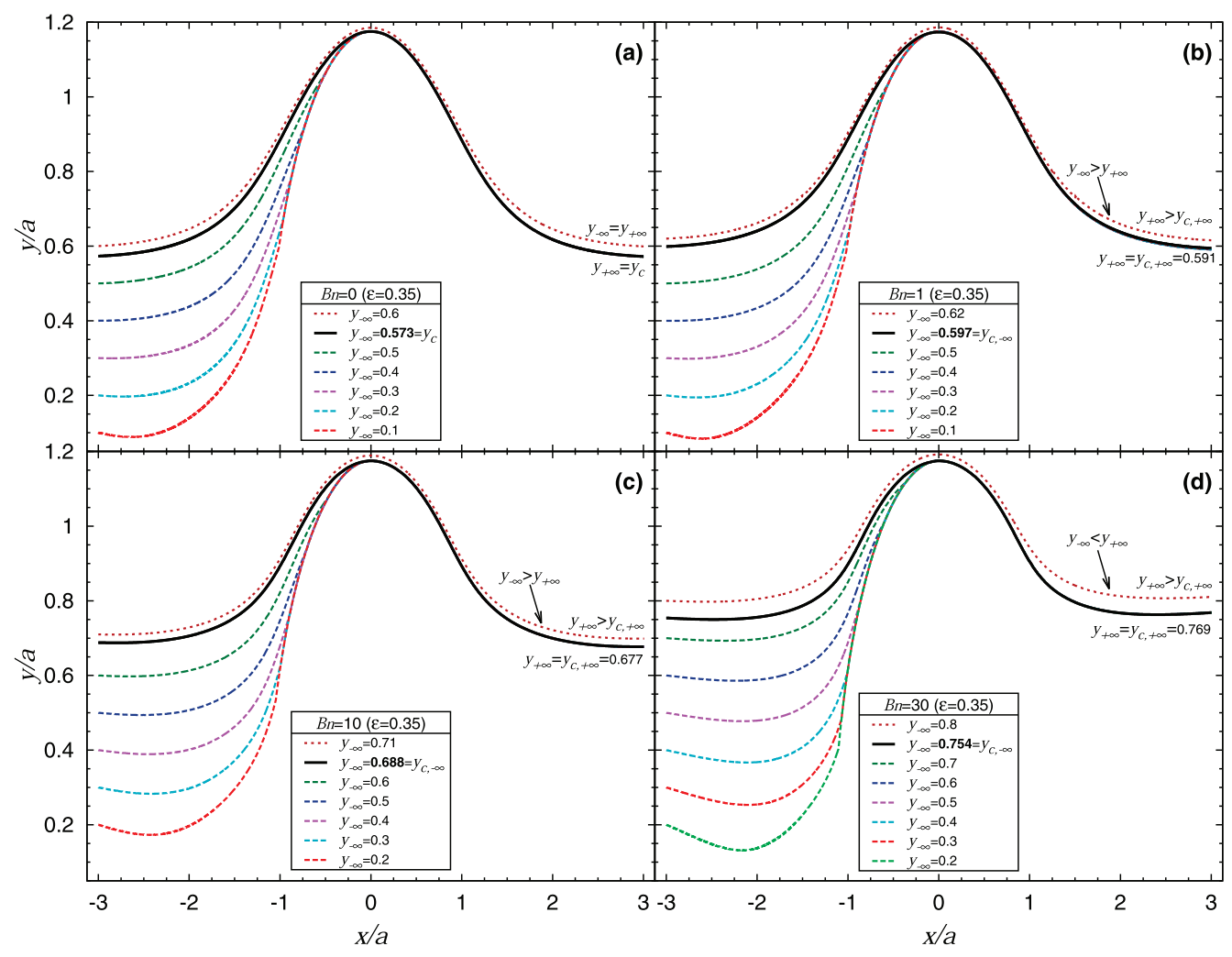

Fig. 19. Bingham effect: trajectories with varying initial offset for the roughness parameter $\varepsilon=0.35$ and different Bingham number Bn.

more convenient and having only one parameter, we thus show only results obtained with this model.

We now discuss the impact of roughness on the trajectories. Fig. 9 (top panel) shows the trajectories obtained in a Newtonian fluid for an initial position $y_{-\infty}=0.3$ for various surface roughness $\varepsilon$. For $\varepsilon \leq 0.2, y_{+\infty}=y_{-\infty}$, symmetric trajectories are observed. This is due to the fact that particles do not touch, as the minimum interparticle distance in the absence of roughness is slightly higher than 0.2 (see Fig. 6). For $\varepsilon>0.2$, particles come into contact and trajectories are no more symmetric; $y_{+\infty}>y_{-\infty}$, and $y_{+\infty}$ increases as the roughness is increased.

In Fig. 9 (bottom panel), we plot the trajectories in a Newtonian fluid $(B n=0)$ for a surface roughness $\varepsilon=0.35$ and various initial positions $y_{-\infty}$. It is observed that it exists a critical value of the initial offset, denoted $y_{c,-\infty}$ in the sequel, above which trajectories are symmetric, whereas they are asymmetric for $y_{-\infty}<y_{c,-\infty}$. Again, this is due to the fact that particles do not touch when the minimum interparticle distance in the absence of roughness is higher than $\varepsilon$; from Fig. 6, it is seen that it is the case for $y_{-\infty}$ larger than $\simeq 0.55$ for $\varepsilon=0.35$ i.e $y_{c,-\infty}(\varepsilon=0.35) \simeq$ 0.55 . More generally, for any surface roughness $\varepsilon, y_{c,-\infty}(\varepsilon)$ is solution of the equation

$\xi_{\min }\left(y_{c,-\infty}(\varepsilon)\right)=\varepsilon$

Using a bisection method we show that $y_{c,-\infty}=0,573$ for $\varepsilon=0,35$. The critical initial offset $y_{c,-\infty}(\varepsilon)$ is equal to the final offset $y_{+\infty}$ for all the trajectories with initial offset lower than $y_{c,-\infty}(\varepsilon)$. This is the direct consequence of (i) fore-aft symmetry in Newtonian fluids in the absence of inertia, and (ii) the da Cunha and Hinch [12] model used in this work. Indeed, when particles come into contact, they stay at their minimum separation distance $(\varepsilon)$ as long as they are in the compressive quadrant $\left(y>0, x<0\right.$, for particle $\left.P_{1}\right)$. As soon as the particles enter the extensional quadrant (now $x>0$ for $y>0$ for particle $P_{1}$ ), they interact only hydrodynamically and their trajectory is that of smooth particles experiencing a minimal surface separation equal to $\varepsilon$. We checked in our simulations that the functions $y_{c,-\infty}(\varepsilon)$ and $\xi_{\min }\left(y_{-\infty}\right)$ are identical (see Fig. 10), as already shown in $[23,50]$.

\subsection{Impact of the Bingham number}

\subsubsection{Hydrodynamic interactions}

We plot the trajectories of particle $P_{1}$ for given Bingham numbers $B n$ and various initial offset $y_{-\infty}$, for purely hydrodynamic interactions $(\varepsilon=0)$ in Fig. 11.

Several trends can be observed. First, increasing the Bingham number seems to cause the disappearance of closed trajectories. This is correlated to the observation of reversing trajectories for a wider range of initial positions when increasing $B n$. E.g., for $y_{-\infty}=0.1$, open trajectories are observed for $B n=0$ and 1, a closed trajectory is observed for $B n=10$, while a reversing trajectory is observed for $B n=30$. An other related observation is that, for large $B n$, particles tend to move toward the $\mathrm{x}$-axis when approaching and to move away after crossing. All of these trends have also been observed in another nonlinear problem by Kulkarni and Morris [38], for inertial flows. However, as expected in the absence of inertia, fore-aft symmetry of particles trajectories is observed here in all cases. This last observation is in contradiction with experimental observations of fore-aft asymmetry in a paper under review at the same time as our paper [21]; in yield stress fluids such as concentrated microgel suspensions, important elastic effects - absent in our simulations - may indeed lead to fore-aft asymmetry [22].

We now plot in Fig. 12 the particle P1 trajectories (left) and the interparticle distance $\xi$ vs particle P1 horizontal position (right) for varying Bingham numbers. It can be seen that at large $B n$ the particles first approach without significant hydrodynamic interaction: indeed the straight part of the $\xi(x)$ curves corresponds to two approaching particles that would move respectively along horizontal streamlines $y(x)=y_{-\infty}$ and $y(-x)=-y_{-\infty}$. The departure from this flow driven motion to a regime in which interparticle hydrodynamic interaction affects the particle trajectories depends on $B n$ : increasing the Bingham number seems 


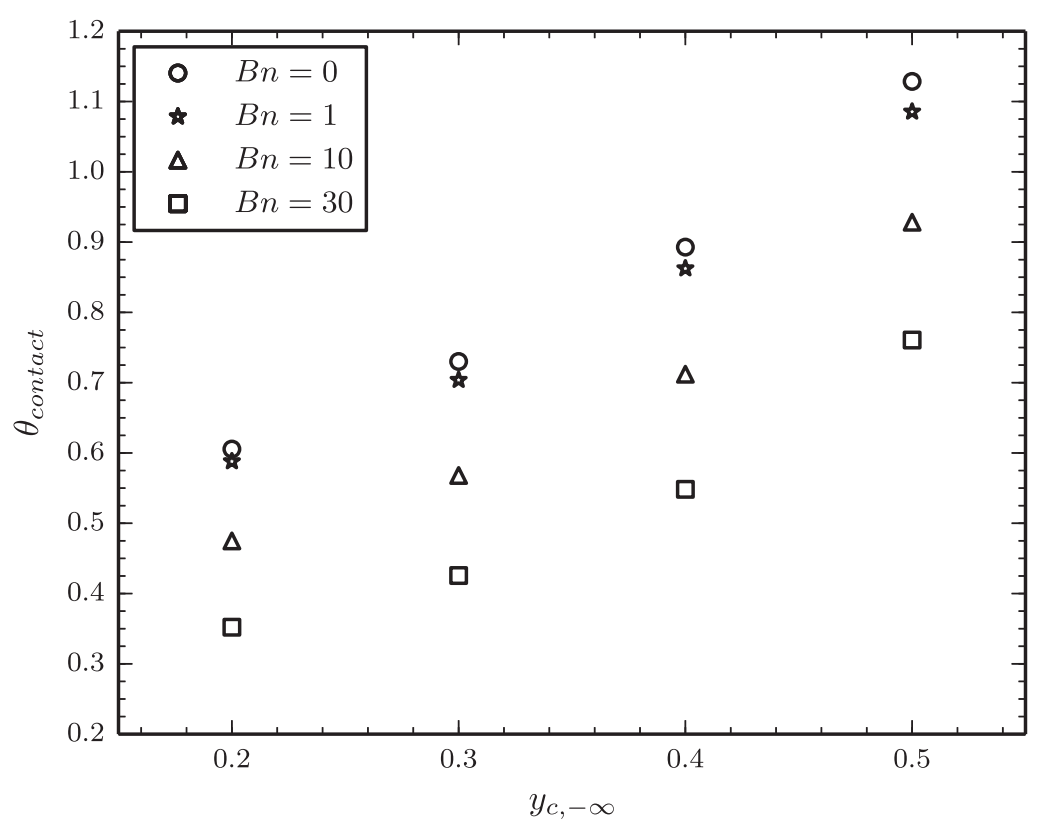

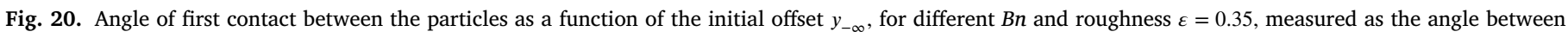
the two particle centers and the $O x$ axis. The angle of last contact is equal to $\pi / 2$ whatever the roughness, the initial position and the Bingham number.

to decrease the range of interparticle hydrodynamic interaction. This is consistent with the recent experimental observations of Firouznia et al. [21] for particles trajectories in a Carbopol gel. It is reminded that the slight departure from symmetry in Fig. 12 (left) provides an estimate of the numerical error in our simulations, which is increased as $B n$ is increased; for this reason, we do not go beyond $B n=30$ in this work.

Closer inspection of the trajectories (Fig. 12 - left) and of the $\xi(x)$ functions (Fig. 12 - right) shows that the particles come closer on to the other when $B n$ is increased. As it is clearly seen in bottom Fig. 12 for $y_{-\infty}=0.2$, at $B n=0$ particles smoothly approach each other while at $B n=30$ there are two regimes: for most interparticle distances they hardly interact while they suddenly repel strongly when the interparticle distance decreases to approximatively 0.15. During all their hydrodynamic interaction, this distance does not change significantly. To measure this effect it is convenient to introduce a critical distance $\xi_{\text {cri }}$ as the gap between the particles at which departure from flow driven motion occurs. More precisely, as a single particle suspended in a large volume of fluid undergoing a simple sheared fluid moves in a horizontal straight line, departure from the rectilinear motion is the signature of the presence in the neighborhood of one or several others particles. We arbitrarily define $\xi_{c r i}$ as the distance between particles at which the gap between straight horizontal trajectories and real trajectories become larger than $5 \%$ of the particle radius. The critical distance $\xi_{\text {cri }}$ is clearly an decreasing function of the Bingham number, which is reminiscent to what is observed for the creeping motion of a solid object in a yield stress fluid [4,54]. To illustrate this, we plot in Fig. 13 the interparticle distance of departure from the flow driven motion $\xi_{c r i}$ (estimated to occur for a gap between straight trajectories and real trajectories smaller than $5 \%$ of the particle radius) as a function of the Bingham number.

As a consequence the minimal separation distance $\xi_{\min }$ between two smooth particles decreases when $B n$ increases (see Fig. 14) which in turn enhances the surface roughness effects: contact between particles should thus occur for smaller surface roughness at large $\mathrm{Bn}$. This point is further discussed in the next section.

Understanding the hydrodynamic interaction between particles requires to study the velocity and shear rates fields around particles. To do so, we plot the colormap of shear rate around one particle (left) and two particles (right) in linear shear flow of a Newtonian fluid $(B n=0)$ in Fig. 15 and in linear flow of a Bingham fluid $(B n=30)$ in Fig. 16.
It is readily seen from Figs. 15 and 16 that the shear rate fields for $B n=0$ and $B n=30$ are very different, which explains why the hydrodynamic interactions are also different. Transitions from high shear rate regions to low shear rate regions are more smooth for the Newtonian fluid than for the Bingham fluid, which is not surprising since it is well known that plastic materials are more prone to localization than viscous ones. Moreover the maximum value of the shear rate is larger for Bingham fluid than for Newtonian fluid. This is also a direct consequence of stronger localization of shear rate for plastic material. Furthermore a "squared" rigid region appears around the particle when the Bingham number is large $(B n=30)$ as it can be seen in Fig. 16 .

Because of the strong differences between the Newtonian and Binghamian shear rate field morphologies it is difficult to assess the relative magnitude of the hydrodynamic interaction from Figs. 15 and 16 even it is clearly seen that the flow is strongly modified in the area between the particles. A convenient quantitative evaluation of hydrodynamic interaction ranges has been proposed by [21]. As they did, we compute the normalized norm of the difference between the computed velocity field and the far field velocity (ie the quantity $\left\|u-\dot{\gamma} y e_{x}\right\| \mid / \max \|u\|$ ) over the domain $\Omega \backslash P(t)$. Variation of normalized disturbance velocity around one particle at fixed distance $r / a=2$ from the particle center is plotted in Fig. 17 and the same quantity as a function of the particle center distance along the $\theta=45^{\circ}$ direction is represented in Fig. 18. It is readily seen in Figs. 17 and 18 that the relative disturbance velocity decays more rapidly for viscoplastic flow. Moreover, similarly to what has been observed by [21], the relative disturbance velocity norm is maximum in the $\theta=45^{\circ}$ direction for a Newtonian fluid whereas it is no more the case when the Bingham number is large enough. When comparing our results to the experimental work of [21], It should be kept in mind that our simulations have been performed for a $2 \mathrm{~d}$ system (circular cylinders) whereas experimental data are for a $3 \mathrm{~d}$ system (spheres).

\subsubsection{Impact of roughness}

We now study the case of rough particles.

In Fig. 19, we plot the trajectories of particles suspended in a Bingham fluid for a surface roughness $\varepsilon=0.35$ and various initial positions $y_{-\infty}$, for various $B n$. As for a Newtonian fluid $(B n=0)$, it exists a critical offset $y_{c,-\infty}$ below which all trajectories lead to a contact and follow 
the same trajectory in the extensional quadrant, leading to a depletion zone; again, $y_{c,-\infty}(\varepsilon, B n)$ and $\xi_{\min }\left(y_{-\infty}, B n\right)$ are identical (see Fig. 14). All the trajectories starting from $y_{-\infty} \leq y_{c}(\varepsilon, B n)$ reach the same final offset $y_{c}(\varepsilon, B n)$ while particles starting from $y_{-\infty}>y_{c}(\varepsilon, B n)$ do not experience direct contact during their crossing. $y_{c}(\varepsilon, B n)$ is an increasing function of $B n$. In other words, there is a fore-aft asymmetry which is enhanced by plastic effects; this feature is also observed in recent experiments [21]. Another related feature is that contacts between particles occur for smaller surface roughness at large Bn. This is illustrated in Fig. 14 .

In addition to the enhanced fore-aft asymmetry, increasing the Bingham number leads to longer contact duration. To characterize quantitatively this feature, we plot the angle of first contact between the particles as a function of the initial offset $y_{-\infty}$, for different $B n$ and roughness $\varepsilon=0.35$ in Fig. 20. The contact angle is defined as the angle between the two particle centers and the $O x$ axis. It is reminded that for our contact model, the angle of last contact is equal to $\pi / 2$ whatever the roughness, the initial position and the Bingham number; therefore, the smaller the value of the first contact angle, the longer the contact duration. This is likely related to the observation of smaller ranges of hydrodynamic interaction between particles for large Bingham numbers (see Fig. 13).

These observations of Bingham-number-dependent fore aftasymmetry and contact duration must have an impact on the microstructure of suspensions of particles in a yield stress fluid. Consistently, a large impact of the Bingham number was reported on the microstructure of concentrated suspensions in Ovarlez et al. [45]: the shear-rate-dependent depletion zones observed through X-ray tomography for real suspensions can at least qualitatively be explained by the enhanced fore-aft asymmetry of the two particles trajectories at large Bingham number.

\section{Conclusion}

We have studied numerically the pair trajectories of rigid circular particles in a two dimensional inertialess simple shear flow of a (Binghamian) yield stress fluid. Due to the nonlinear behavior of the suspending fluid, it is found that the trajectories of the particles depend on the Bingham number. In the absence of interparticle contacts, fore-aft symmetry is observed in all cases; however, the particles are found to come closer to each other as the Bingham number is increased. When particles come into contact, due to the particle roughness, a fore-aft asymmetry of trajectories is observed. Plastic effects are found to enhance surface roughness effects: contacts between particles occur for smaller surface roughness at large $\mathrm{Bn}$. Moreover, the magnitude of the asymmetry is an increasing function of both the Bingham number and the particle roughness. The impact of the Bingham number $B n$ on the trajectory is likely related to a decrease of the range of hydrodynamic interactions as $B n$ is increased. These observations may explain why the microstructure of suspensions of particles in a yield stress fluid is shear-rate-dependent [45] leading to a complex nonlinear macroscopic behavior.

\section{References}

[1] C. Ancey, H. Jorrot, Yield stress for particle suspensions within a clay dispersion, J. Rheol. 45 (2) (2001) 297-319, doi:10.1122/1.1343879.

[2] A.M. Ardekani, R. Rangel, Numerical investigation of particle-particle and particle-wall collisions in a viscous fluid, J. Fluid Mech. 596 (2008) 437-466, doi:10.1017/S0022112007009688.

[3] R. Avazmohammadi, P.P. Castaneda, Macroscopic rheological behavior of suspensions of soft solid particles in yield stress fluids, J. Non-Newton. Fluid Mech. 234 (2016) 139-161, doi:10.1016/j.jnnfm.2016.05.005.

[4] A.N. Beris, J.A. Tsamopoulos, R.C. Armstrong, R.A. Brown, Creep motion of a sphere trhrough a bingham plastic, J. Fluid Mech. 158 (1985) 219-244, doi:10.1017/S0022112085002622.

[5] F. Blanc, E. Lemaire, A. Meunier, F. Peters, Microstructure in sheared non-brownian concentrated suspensions, J. Rheol. 57 (1) (2013) 273-292, doi:10.1122/1.4766597.

[6] F. Blanc, F. Peters, E. Lemaire, Experimental signature of the pair trajectories of rough spheres in the shear-inducednduced microstructure in noncolloidal suspensions, Phys. Rev. Lett. 107 (20) (2011) 208302, doi:10.1103/PhysRevLett.107.208302.
[7] J.F. Brady, J.F. Morris, Microstructure of strongly sheared suspensions, and its impact on rheology and diffusion, J. Fluid Mech. 348 (1997) 103-139, doi:10.1017/S0022112097006320.

[8] X. Chateau, G. Ovarlez, K.-L. Trung, Homogenization approach to the behavior of suspensions of noncolloidal particles in yield stress fluids, J. Rheol. 52 (2) (2008) 489-506, doi:10.1122/1.2838254.

[9] Y.J. Choi, M.A. Hulsen, H.E.H. Meijer, An extended finite element method for the simulation of particulate viscoelastic flows, J. Non-Newt. Fluid Mech. 165 (1112) (2010) 607-624, doi:10.1016/j.jnnfm.2010.02.021.

[10] S. Court, M. Fournié, A. Lozinski, A fictitious domain approach for the stokes problem based on the extended finite element method, Int. J. Numer. Meth. Fluids 74 (2) (2014) 73-99, doi:10.1002/fld.3839.

[11] P. Coussot, Mudflow Rheology and Dynamics, Taylor \& Francis, 1997.

[12] F.R. da Cunha, E.J. Hinch, Shear-induced dispersion in a dilute suspension of rough spheres, J. Fluid. Mech. 309 (1996) 211-223, doi:10.1017/S0022112096001619.

[13] S. Dagois-Bohy, S. Hormozi, E. Guazzelli, O. Pouliquen, Rheology of dense suspensions of non-colloidal spheres in yield-stress fluids, J. Fluid Mech. 776 (2015), doi:10.1017/jfm.2015.329.

[14] S.L. Dance, E. Climent, M.R. Maxey, Collision barrier effects on the bulk flow in a random suspension, Phys. Fluids 16 (3) (2004) 828-831, doi:10.1063/1.1637349.

[15] G. D'Avino, P.L. Maffetone, Particle dynamics in viscoelastic liquids, J. Non-Newton. Fluid Mech. 215 (2015) 80-104, doi:10.1016/j.jnnfm.2014.09.014.

[16] R.H. Davis, Effects of surface roughness on a sphere sedimenting through a dilute suspension of neutrally buoyant spheres, Phys. Fluids A 4 (12) (1992) 2607-2619, doi:10.1063/1.858450.

[17] E.J. Dean, R. Glowinski, Operator-splitting methods for the simulation of Bingham visco-plastic flow, Chin. Ann. Math. 23 (2) (2002) 187-204, doi:10.1142/S0252959902000183.

[18] E.J. Dean, R. Glowinski, G. Guidoboni, On the numerical simulation of Bingham visco-plastic flow: old and new results, J. Non-Newton. Fluid Mech. 142 (2007) 3662, doi:10.1016/j.jnnfm.2006.09.002.

[19] G. Duvaut, J.L. Lions, Inequalities in Mechanics and Physics, Springer, Berlin, 1976

[20] T.S. Erdogan, Determination of aggregate Shape Properties Using X-Ray Tomographic Methods and the Effect of Shape on Concrete Rheology, Ph.D. thesis, University of Texas at Austin, 2005.

[21] M. Firouznia, B. Metzger, G. Ovarlez, S. Hormozi, The hydrodynamic interaction of two spherical particles in simple-shear flows of yield stress fluid, J. Non-Newton. Fluid Mech 255 (2018) 19-38, doi:10.1016/j.jnnfm.2018.03.006.

[22] D. Fraggedakis, Y. Dimakopoulos, J. Tsamopoulos, Yielding the yield-stress analysis: a study focused on the effects of elasticity on the settling of a single spherical particle in simple yield-stress fluids, Soft Matter 12 (24) (2016) 5378-5401, doi:10.1039/c6sm00480f.

[23] J. Frechette, G. Drazer, Directional locking and deterministic separation in periodic arrays, J. Fluid Mech. 627 (2009) 379-401, doi:10.1017/S0022112009005941.

[24] I.A. Frigaard, C. Nouar, On the usage of viscosity regularisation methods for viscoplastic fluid flow computation, J. Non-Newton. Fluid Mech. 127 (1) (2005) 1-26, doi:10.1016/j.jnnfm.2005.01.003.

[25] M.R. Geiker, M. Brandl, L.N. Thrane, L.F. Nielsen, On the effect of coarse aggregate fraction and shape on the rheological properties of self-compacting concrete, Cem. Concr. Aggreg. 24 (1) (2002) 3-6.

[26] R. Glowinski, T.-W. Pan, T.I. Hesla, D.D. Joseph, A distributed lagrange multiplier/fictitious domain method for particulate flows, Int. J. Multiph. Flow 25 (1999) 755-794, doi:10.1016/S0301-9322(98)00048-2.

[27] R. Glowinski, T.-W. Pan, T.I. Hesla, D.D. Joseph, P. J., A fictitious domain approach to the direct numerical simulation of incompressible viscous flow past moving rigid bodies: application to particulate flow, J. Comput. Phys. 169 (2001) 363426, doi:10.1006/jcph.2000.6542.

[28] R. Glowinski, T.-W. Pan, J. Periaux, A lagrange multiplier/fictitious domain method for the dirichlet problem - generalization to some flow problems, Japan J. Indust. Appl. Math. 12 (1) (1995) 87-108, doi:10.1007/BF03167383.

[29] R. Glowinski, T.-W. Pan, J. Periaux, A lagrange multiplier/fictitious domain method for the numerical simulation of incompressible viscous flow around moving rigid bodies: (i) case where the rigid body motions are known a priori, C. R. Acad. Sci. Series I - Mathematics 324 (3) (1997) 361-369.

[30] E. Guazzelli, J.F. Morris, A Physical Introduction to Suspension Dynamics, Cambridge University Pres, 2012.

[31] M. Haddadi, J.F. Morris, Topology of pair-sphere trajectories in finite inertia suspension shear flow and its effects on microstructure and rheology, Phys. Fluids 27 (4) (2015), doi:10.1063/1.4917030.

[32] H.H. Hu, Direct simulation of flows of solid-liquid mixtures, Int. J. Multiphase Flow 22 (2) (1996) 335-352, doi:10.1016/0301-9322(95)00068-2.

[33] H.H. Hu, D.D. Joseph, M. Crochet, Direct simulation of fluid particle motions, Theor. Comput. Fluid. Dyn. 3 (5) (1992) 285-306, doi:10.1007/BF00717645.

[34] H.H. Hu, N.A. Patankar, M.Y. Zhu, Direct numerical simulations of fluid-solid systems using the arbitrary lagrangian-eulerian technique, J. Comput. Phys. 169 (2) (2001) 427-462, doi:10.1006/jcph.2000.6592.

[35] A.A. Johnson, T.E. Tezduyar, Simulation of multiple spheres falling in a liquidfilled tube, Comput. Methods Appl. Mech. Engrg. 134 (3-4) (1996) 351-373, doi:10.1016/0045-7825(95)00988-4.

[36] Y.M. Joumana, M. Chaouche, M. Guerinet, M. Moranville, N. Roussel, From ordinary rheology concrete to self compacting concrete: a transition between frictional and hydrodynamic interactions, Cem. Concr. Res. 38 (2008) 890-896.

[37] T. Kempe, J. Fröhlich, An improved immersed boundary method with direct forcing for the simulation of particle laden flows, J. Comput. Phys. 231 (9) (2012) 36633684, doi:10.1016/j.jcp.2012.01.021. 
[38] P.M. Kulkarni, J.F. Morris, Pair-sphere trajectories in finite-reynolds-number shear flow, J. Fluid Mech. 596 (2008) 413-435, doi:10.1017/S0022112007009627.

[39] F. Mahaut, X. Chateau, P. Coussot, G. Ovarlez, Yield stress and elastic modulus of suspensions of noncolloidal particles in yield stress fluids, J. Rheol. 52 (1) (2008) 287-313, doi:10.1122/1.2798234.

[40] F. Mahaut, S. Mokeddem, X. Chateau, N. Roussel, G. Ovarlez, Effect of coarse particle volume fraction on the yield stress and thixotropy of cementitious materials, Cem. Concr. Res. 38 (11) (2008) 1276-1285, doi:10.1016/j.cemconres.2008.06.001.

[41] B. Maury, A many-body lubrication model, Comptes Rendus Acad. Sci. - Series I Mathematics 325 (9) (1997) 1053-1058, doi:10.1016/S0764-4442(97)89104-5.

[42] B. Maury, A time-stepping scheme for inelastic collisions, Numer. Math. 102 (4) (2006) 649-679, doi:10.1007/s00211-005-0666-6.

[43] J.F. Morris, A review of microstructure in concentrated suspensions and its implications for rheology and bulk flow, Rheol. Acta 48 (2009) 909-923, doi:10.1007/s00397-009-0352-1.

[44] N.-Q. Nguyen, A.J.C. Ladd, Lubrication corrections for lattice-boltzmann simulations of particle suspensions, Phys. Rev. E 66 (2002) 046708, doi:10.1103/PhysRevE.66.046708.

[45] G. Ovarlez, F. Mahaut, S. Deboeuf, N. Lenoir, S. Hormozi, X. Chateau, Flows of suspensions of particles in yield stress fluids, J. Rheol. 59 (2015) 1449, doi:10.1122/1.4934363.

[46] G. Ovarlez, F.B. P., Coussot, X. Chateau, Shear-induced sedimentation in yield stress fluids, J. Non-Newton. Fluid Mech. 177-178 (2012) 19-28, doi:10.1016/j.jnnfm.2012.03.013.

[47] R. Papanastasiou, Flows of material with yield, J. Rheol. 31 (1987) 385, doi:10.1122/1.549926.

[48] N.A. Patankar, P. Singh, D.D. Joseph, R. Glowinski, T.-W. Pan, A new formulation of the distributed lagrange multiplier/fictitious domain method for particulate flows, Int. J. Multiphase Flow 26 (9) (2000) 1509-1524, doi:10.1016/S0301-9322(99)00100-7.

[49] I. Rampall, J.R. Smart, D.T. Leighton, The influence of surface roughness on the particle-pair distribution function of dilute suspensions of noncolloidal spheres in simple shear flow, J. Fluid Mech. 339 (1997) 1-24, doi:10.1017/S002211209600479X.
[50] S.R. Risbud, G. Drazer, Trajectory and distribution of suspended non-brownian particles moving past a fixed spherical or cylindrical obstacle, J. Fluid Mech. 714 (2013) 213-237, doi:10.1017/jfm.2012.468.

[51] F. Snijkers, R. Pasquino, J. Vermant, Hydrodynamic interactions between two equally sized spheres in viscoelastic fluids in shear flow, Langmuir 29 (19) (2013) 5701-5713, doi:10.1021/la4006604.

[52] M. Sommerfeld, Validation of a stochastic lagrangian modelling approach for interparticle collisions in homogeneous isotropic turbulence, Int. J. Multiphase Flow 27 (10) (2001) 1829-1858, doi:10.1016/S0301-9322(01)00035-0.

[53] H. Tabuteau, J.C. Baudez, X. Chateau, P. Coussot, Flow of a yield stress fluid over a rotating surface, Rheol. Acta 46 (3) (2007) 341-355, doi:10.1007/s00397-006-0125-z.

[54] D.L. Tokpavi, P. Jay, A. Magnin, L. Jossic, Experimental study of the very slow flow of a yield stress fluid around a circular cylinder, J. Non-Newt. Fluid Mech. 164 (2009) 35-44, doi:10.1016/j.jnnfm.2009.08.002.

[55] Z. Toutou, N. Roussel, Multi scale experimental study of concrete rheology: from water scale to gravel scale, Mater. Struct. 39 (2006) 189-199, doi:10.1617/s11527-005-9047-y.

[56] K. Usman, Numerical Analysis of Collision Models in 2D Particulate Flow, Ph.D. thesis, TU Dortmund, 2013.

[57] T.S. Vu, G. Ovarlez, X. Chateau, Macroscopic behavior of bidisperse suspensions of noncolloidal particles in yield stress fluids, J. Rheol. 54 (4) (2010) 815-833, doi:10.1122/1.3439731.

[58] D. Wan, S. Turek, Fictitious boundary and moving mesh methods for the numerical simulation of rigid particulate flows, J. Comput. Phys. 222 (1) (2007) 28-56, doi:10.1016/j.jcp.2006.06.002.

[59] H.J. Wilson, R.H. Davis, The viscosity of a dilute suspension of rough spheres, J. Fluid Mech. 421 (2000) 339-367, doi:10.1017/S0022112000001695.

[60] H.J. Wilson, R.H. Davis, Shear stress of a monolayer of rough spheres, J. Fluid Mech. 452 (2002) 425-441, doi:10.1017/S0022112001006838.

[61] J. Xu, R. Glowinski, Numerical methods for non-Newtonian fluids, Handbook of Numerical Analysis, vol. 16, Elsevier, Amsterdam, 2011. 